\title{
talus et soutènements en dynamique des sols
}

\section{dynamic behaviour and design of slopes and retaining structures}

\author{
F. SCHLOSSER \\ Professeur à l'École Nationale des Ponts et Chaussées*, \\ Directeur de Terrasol \\ L. DORMIEUX \\ École Nationale des Ponts et Chaussées (C.E.R.M.E.S.)*
}

\section{Résumé}

La connaissance du comportement des talus et des ouvrages de soutènement sous l'action d'un séisme a beaucoup progressé depuis une vingtaine d'années. Ce rapport présente un état de connaissances sur le thème, divisé en trois parties: un exposé des résultats les plus récents sur le comportement dynamique des sols saturés, notamment les influences de la contrainte initiale et de la structure initiale sur la liquéfaction des sables (phénomène de déformation d'écoulement); une présentation des principales méthodes de dimensionnement des talus de barrages et des pentes naturelles; un point sur le comportement des murs-poids et sur l'évolution des méthodes de dimensionnement, avec le cas récent des murs en terre armée. On montre qu'il convient d'être prudent avec les modèles réduits et que l'inertie de l'ouvrage joue un rôle essentiel. Une liste assez exhaustive de références bibliographiques récentes accompagne le rapport.

\begin{abstract}
Knowledge of the behaviour of slopes and retaining structures under a seismic loading has largely increased during the last twenty years. A corresponding state-ofthe-art is presented in this report which is divided into three parts : an indication of the most recent results on the dynamic behaviour of saturated soils, including the influences on liquefaction of sand of the initial shear stress and of the initial grain arrangement (flow deformation phenomenon); a review of the main design methods for embankment slopes and natural slopes; a presentation of the behaviour of the gravity retaining walls and of the evolution of the design methods, with a brief description of the seismic design of Reinforced Earth walls. It is shown that reduced scale models constitute a limited tool and that the inertia of the structure has a great effect. Comprehensive recent references on each topic are listed.
\end{abstract}




\section{INTRODUCTION}

L'effet des séismes sur les pentes et sur les ouvrages de soutènement a fait, depuis de nombreuses années et notamment depuis vingt ans, l'objet d'observations et d'études qui ont conduit à toute une panoplie de méthodes de dimensionnement. Pour autant, beaucoup de questions restent en suspens, et certains types d'ouvrages de soutènement, ainsi que les pentes naturelles d'une façon générale, n'ont été que très peu abordés.

Dans le domaine des pentes, les études ont été principalement motivées par la construction des barrages en terre en zone sismique. Il s'agit donc avant tout de pentes de remblais dans des conditions d'homogénéité très différentes de celles des pentes naturelles. Après les premières utilisations de la méthode pseudostatique par TERZAGHI en 1950, les deux principaux apports qui ont marqué les développements dans ce domaine sont :

- N.M. NEWMARK. 5e Conférence Rankine (1965).

- H.B. SEED. 19e Conférence Rankine (1979).

Dans le domaine des ouvrages de soutènement, l'origine des études est ancienne et remonte aux classiques travaux de MONONOBE et OKABE sur la poussée dynamique en 1929. Parmi les synthèses les plus intéressantes consacrées, en totalité ou en partie, à ce thème, on doit citer :

- H.B. SEED et R.V. WHITMAN. ASCE Specialty Conference. Ithaca (1970).

- H. NAZARIAN et A. HADJIAN (1979).

- R.V. WHITMAN. Rapport Général au Congrès de Lima (1979).

- S. PRAKASH. Rapport Général au Congrès de Missouri-Rolla (1981).

Certains phénomènes tels que la liquéfaction des sables saturés ou le développement de surpressions interstitielles dans les sols argileux, peuvent être rencontrés, au cours de séismes, dans les pentes comme dans les remblais des ouvrages de soutènements. C'est la raison pour laquelle ce rapport comporte un chapitre consacré à certains aspects spécifiques du comportement dynamique des sols, qui méritent d'être mis en lumière compte tenu d'études récentes et de leurs implications dans le dimensionnement des ouvrages en terre.

Chacun des deux chapitres portant sur l'un des types d'ouvrages en terre (pentes ou soutènements) comporte une analyse sommaire du comportement de louvrage et un exposé de l'état actuel des connaissances et des méthodes de dimensionnement.

Ce rapport général est accompagné de trois notes rédigées respectivement par $\mathrm{P}$. LONDE, F. BLONDEAU et M. BASTICK. Elles traitent de trois aspects particuliers à propos desquels des recherches s'avèrent encore nécessaires :

- La résistance au cisaillement dynamique des argiles.
- L'influence d'une contrainte de cisaillement initiale sur la liquéfaction.

- Le comportement et le dimensionnement des ouvrages en terre armée sous séisme.

\section{ASPECTS SPÉCIFIQUES DU COMPORTEMENT DYNAMIQUE DES SOLS}

\subsection{Généralités}

Par rapport au comportement statique, le comportement des sols sous sollicitations cycliques se caractérise par le fait que l'accumulation des cycles peut provoquer de grandes déformations sans rapport avec celles provoquées par un cycle unique. Il en résulte la notion de seuil, illustrée à la figure 1 , séparant un domaine de sollicitations conduisant à un état stabilisé, d'un domaine où les déformations cumulées s'accentuent jusqu'à la rupture (phénomène de rochet). La plupart des résultats obtenus dans les études récentes mettent en évidence l'existence de tels seuils, notamment dans le plan des contraintes principales (courbes ou droites limites) et dans l'espace (p, q,e e) introduit par l'école de Cambridge.

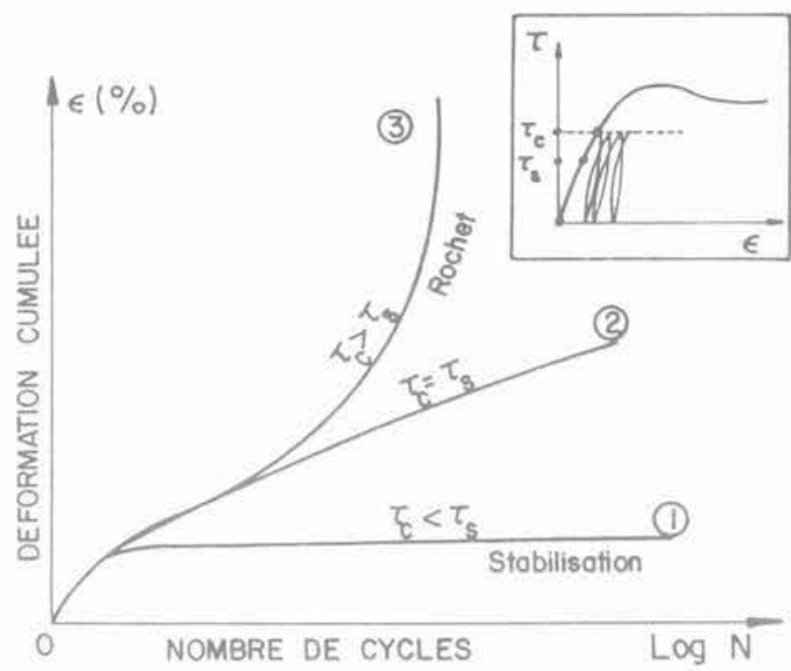

Fig. 1. - Effets cumulés de cycles de chargement sur un sol.

(Essais à la boîte de cisaillement).

La notion de seuil permet donc de caractériser les sollicitations ne conduisant pas à la rupture, même lorsqu'elles sont appliquées durant un nombre élevé de cycles. Toutefois, une sollicitation sismique réelle intervient pendant une durée limitée qui peut être suffisamment courte pour qu'un chargement d'amplitude supérieure au seuil ne provoque que des déformations admissibles. Aussi, utilisé pour caractériser la résistance au cisaillement cyclique, le seuil constitue-t-il en général un critère trop conservatif. En fait, de nombreux auteurs préfèrent relier lamplitude de la sollicitation cyclique $\tau_{\text {c }}$ au nombre de cycles $\mathrm{N}$ requis pour provoquer la rupture définie en général par un taux arbitraire de déformation $\varepsilon_{Y}$. 
A partir de la famille de courbes $\tau_{\text {, }}\left(\mathrm{N}, \varepsilon_{v}\right)$, SEED (1966) relie la contrainte de cisaillement totale $\tau_{t}=\tau_{0}+\tau_{\text {s }}$ (statique + cyclique), conduisant à la rupture en un nombre donné de cycles $\mathrm{N}_{r}$, à la contrainte normale effective $\sigma^{\prime}$, s'exerçant avant sollicitation sur la facette cisaillée, et à l'inclinaison $\tau_{0} / \sigma^{\prime}$ 。 de la contrainte statique initiale sur cette facette. Il obtient ainsi dans le plan de Mohr une famille de droites dépendant de trois paramètres et caractérisant la résistance au cisaillement cyclique :

$$
\tau_{\mathrm{r}}=\mathrm{f}\left(\sigma_{0}^{\prime}, \mathrm{N}_{\mathrm{r}}, \mathcal{E}_{\mathrm{r}}, \tau_{0} / \sigma_{0}^{\prime}\right)
$$

Un tel critère constitue une généralisation du concept de courbe intrinsèque, utilisé classiquement pour les chargements monotones.

Certains aspects majeurs du comportement des sols sous chargement dynamique, qui sont présentés dans la suite, font largement appel à cette définition de la résistance au cisaillement dynamique.

\subsection{Résistance au cisaillement dynamique des argiles}

L'étude de la résistance au cisaillement des argiles saturées soumises à une sollicitation cyclique n'est vraiment devenue fructueuse qu'à dater du moment où l'on a mesuré le développement des surpressions interstitielles au cours d'essais à l'appareil triaxial, ce qui constitue et reste un difficile problème technologique.

D'une façon générale, on peut distinguer deux types de chargements cycliques, pour lesquels le comportement de l'argile est différent. Un chargement cyclique symétrique, dans lequel le sens du cisaillement total (statique + dynamique) s'inverse à chaque cycle, et un chargement cyclique dans lequel le sens de cette contrainte de cisaillement reste constant. Dans le premier cas, pour des cycles uniformes, on observe une augmentation progressive de l'amplitude de la déformation à chaque cycle, amplitude qui contrôle l'apparition éventuelle de la rupture. Dans le deuxième cas, il y a accumulation de la déformation dans la direction du cisaillement et c'est la déformation cumulée qui contrôle alors le comportement.

Cependant, la résistance au cisaillement cyclique apparaît gouvernée par de nombreux facteurs dont les effets n'ont pas été encore complètement étudiés.

Dans le cas des argiles normalement consolidées, on peut considérer que cette résistance ne dépend que du développement des surpressions interstitielles et qu'il n'y a pas de modification de l'angle de frottement effectif $\varnothing^{\prime}$, tel qu'on peut le mesurer au moyen d'essais statiques (SANGREY et al., 1969; WILSON et GREENWOOD, 1974 ; ANDERSEN, 1976; WOOD, 1976 ; HICHER et ELHOSRI, 1981).

Il semble, par contre, qu'il n'en soit pas de même pour les argiles surconsolidées, dans lesquelles un phénomène de fatigue puisse intervenir à la suite d'un grand nombre de cycles (HICHER et ELHOSRI, 1981).
Les développements de surpressions interstitielles correspondent essentiellement à des phases de contractance du squelette dans les argiles normalement consolidées ou légèrement surconsolidées et à des phases de dilatance pour les argiles fortement surconsolidées. Les études ont principalement portées sur les argiles normalement consolidées où la pression interstitielle due aux cycles peut atteindre des valeurs importantes. Ainsi MATSUI et ABE (1981) observent des surpressions atteignant la valeur de la contrainte normale effective initiale, dans un processus rappelant la liquéfaction des sables saturés en condition non drainée. La figure 2 montre le chemin des contraintes effectives dans un tel essai réalisé à l'appareil triaxial (TAKAHASHI et al., 1980). On observe des boucles de dilatance et un dépassement de la droite de rupture dans le domaine en extension. Ce dernier point pourrait provenir d'erreurs de mesures (d'après les auteurs) ou d'un effet de surconsolidation (MATSUI et al., 1980).

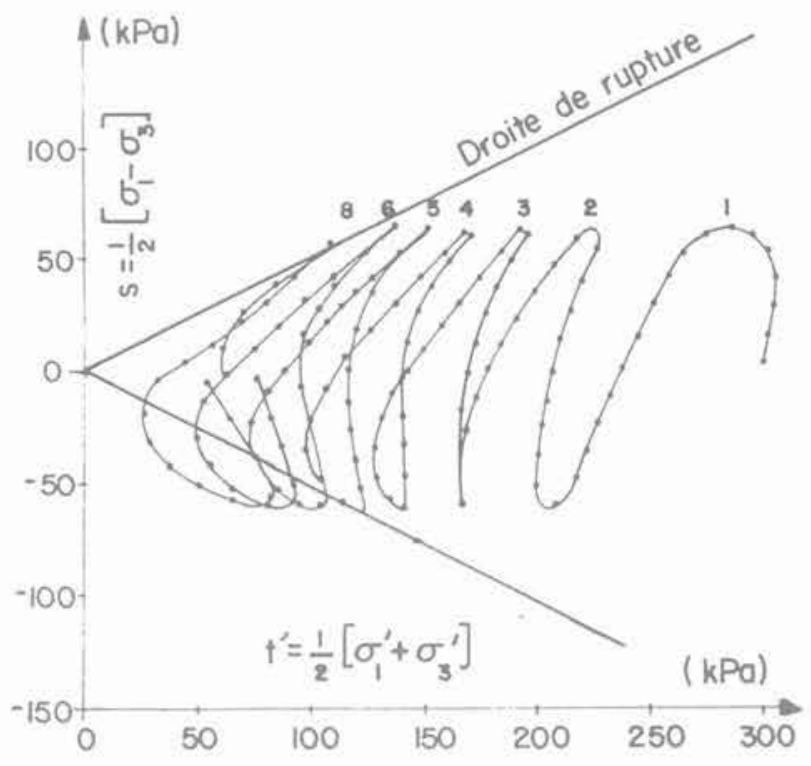

Fig. 2. - Essai de cisaillement cyclique sur une argile molle à l'appareil triaxial.

Chemin en contraintes effectives (TAKAHASHl et al., 1980).

Toujours dans les argiles normalement consolidées, on peut mettre en évidence un seuil pour l'amplitude des cycles de contraintes, seuil en deçà duquel il y a stabilisation des déformations et au-delà duquel on observe un phénomène de rochet. Les figures $3 a$ et $3 b$ (SANGREY et al., 1969) montrent les chemins en contraintes effectives dans chacun des cas précédents et pour des cycles sans inversion du sens du cisaillement. $\mathrm{Ce}$ seuil est généralement déterminé en fonction de la cohésion non drainée $\mathrm{Cu}$. Sa valeur peut varier de $0,3 \mathrm{Cu}$ à $0,8 \mathrm{Cu}$ et dépend entre autres facteurs du type des cycles de chargement (ANDERSEN, 1976) et de la nature minéralogique de l'argile (HICHER et ELHOSRI, 1981). Lorsqu'il y a stabilisation, la valeur limite atteinte par la surpression interstitielle apparait proportionnelle à l'amplitude du cycle (SANGREY et al., 1969) et à la déformation axiale cumulée (WILSON et GREENWOOD, 1974). 

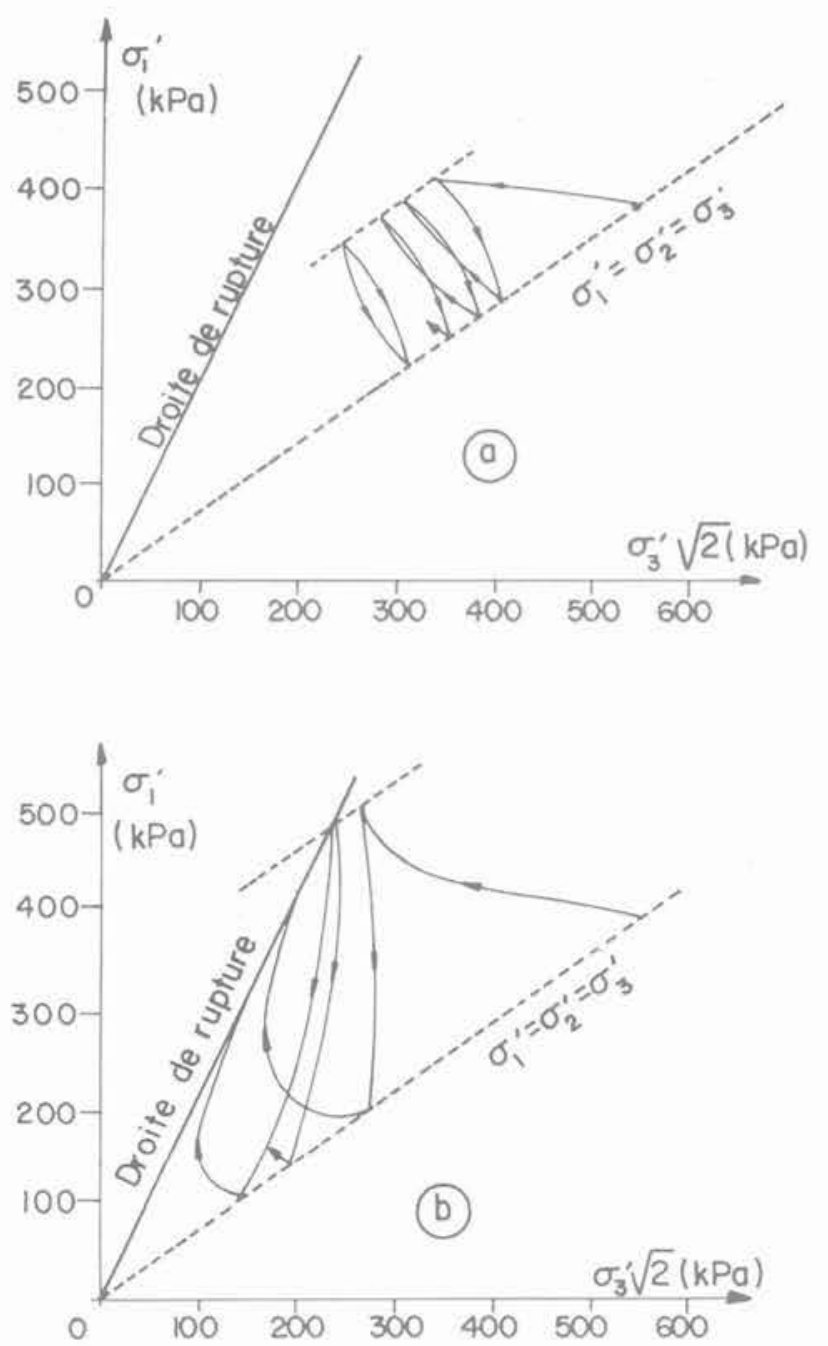

Fig. 3. - Chemins en contraintes effectives dans un chargement cyclique non drainé d'une argile normalement consolidée (SANGREY et al., 1969).

Le développement de surpressions interstitielles positives au cours des cycles de chargement conduit globalement à une diminution de la résistance de l'argile (cohésion $\mathrm{Cu}$ ), telle qu'on peut la mesurer dans un essai de cisaillement non drainé après le chargement cyclique (WOOD, 1976; ANDERSEN, 1976; MATSUI et al., 1980). Cette diminution est très variable. Elle semble même pouvoir dans certains cas être totale (MATSUI et al., 1980). A l'inverse, après dissipation totale des surpressions interstitielles générées par les cycles, l'argile dispose d'une résistance non drainée accrue. Ces deux phénomènes sont visibles sur les résultats de MATSUI et al. (1980) présentés à la figure 4 et dans lesquels a été introduite la notion de rapport de surconsolidation équivalente.

Dans les projets, une étude en laboratoire des développements de la pression interstitielle reste délicate, voire même impossible. C'est la raison pour laquelle on préfère présenter les résultats d'essais de cisaillement cyclique en laboratoire en contraintes totales, en définissant

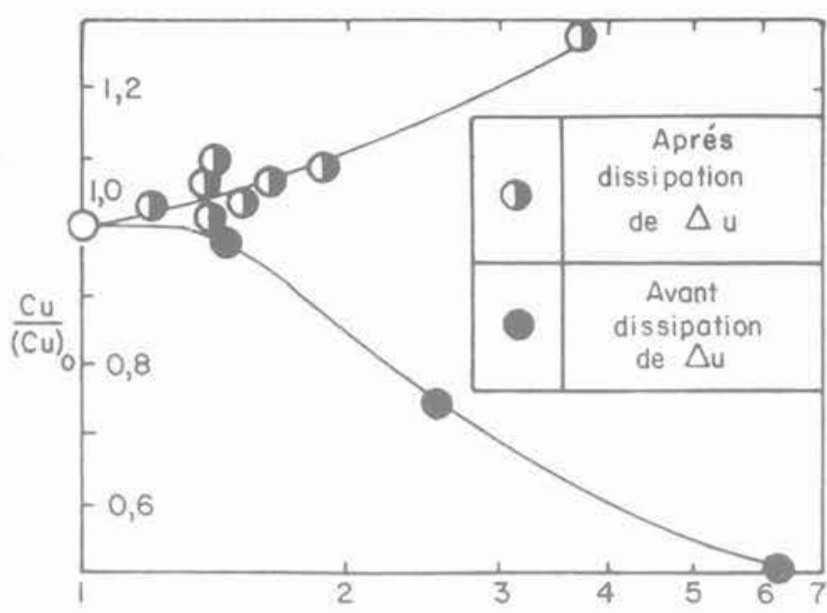

RAPPORT DE SURCONSOLIDATION EQUIVALENT

Fig. 4. - Influence d'un chargement cyclique sur la cohésion non drainée $C_{u}$ (MATSUI et al., 1980).

arbitrairement la rupture par un pourcentage donné de déformation. La figure 5 (ANDERSEN, 1976) donne ainsi la valeur du rapport de la résistance non drainée cyclique (définie pour une déformation de $3 \%$ ) à la cohésion non drainée statique initiale, en fonction du nombre de cycles appliqués.

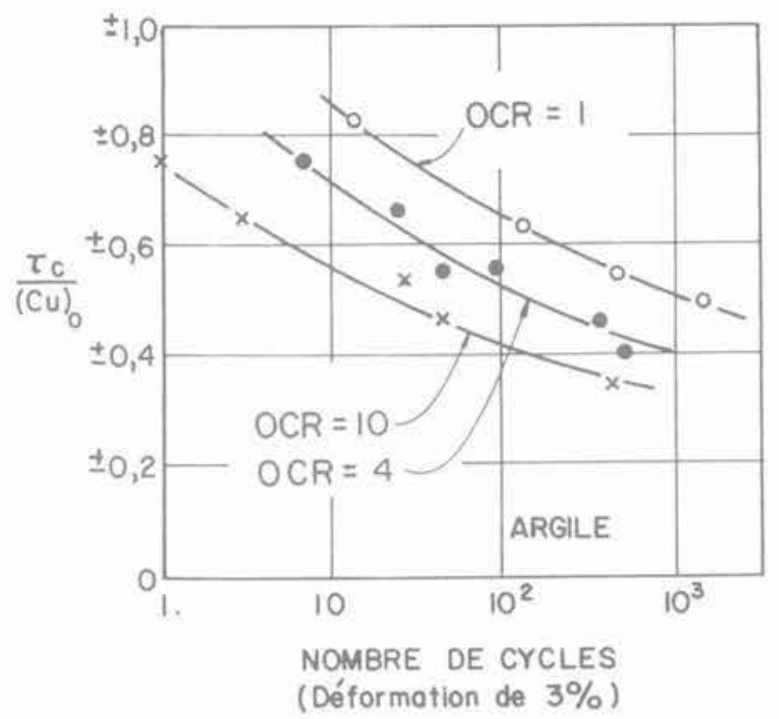

Fig. 5. - Résistance au cisaillement cyclique non drainé d'une argile

(influence de la surconsolidation) (ANDERSEN, 1976).

La vitesse de sollicitation ou la fréquence des cycles constitue un autre facteur important dans la résistance au cisaillement dynamique des argiles. Quelques auteurs l'ont étudié (SEED et CHAN, 1966; MATSUI et al, , 1980). Ce point fait l'objet de la note présentée par P. LONDE à la suite de ce rapport. 


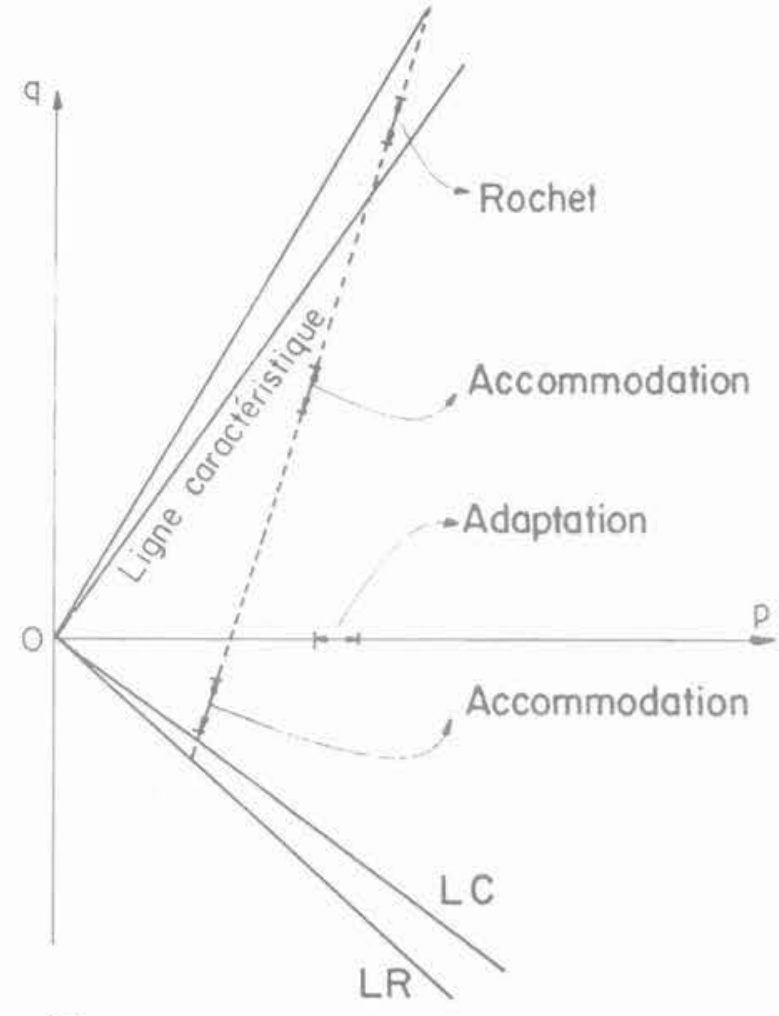

(a)

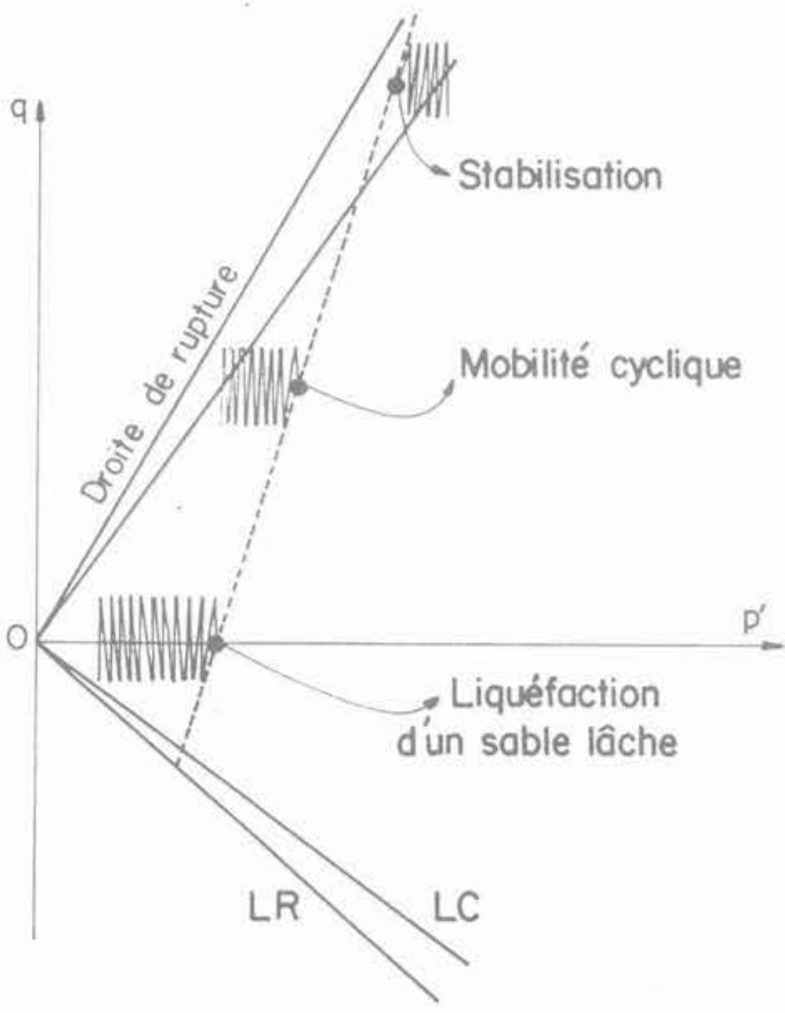

(b)

Fig. 6. - Théorie de la ligne caractéristique (LUONG, 1980).

\subsection{Aspects particuliers du comportement dynamique des sables saturés}

\subsubsection{Traits généraux du comportement}

Le comportement d'un sable saturé dans un chargement cyclique non drainé est essentiellement régi par les surpressions interstitielles qui s'accumulent au cours des cycles. Le développement de surpressions positives ou négatives résulte directement du comportement contractant ou dilatant du squelette durant la sollicitation. Contrairement aux argiles normalement consolidées, un sable peut présenter lors d'un cisaillement cyclique des phases successives de contractance ou de dilatance. Parmi les études les plus récentes, la théorie de l'état caractéristique développée par LUONG (1980), et vérifiée depuis par de nombreux auteurs, met en évidence l'existence de seuils, dans le plan $(p, q)$ des contraintes principales, appelés lignes caractéristiques et séparant le domaine contractant du domaine dilatant. Les figures $6 a$ et $6 b$ s'y réfèrent et présentent les comportements d'un sable en conditions drainée et non drainée.

Les surpressions interstitielles générées dans un sable par un chargement cyclique sont difficiles à prévoir quantitativement car les déformations volumiques du squelette dépendent de nombreux facteurs parmi lesquels la densité relative, l'amplitude du déviateur cyclique, le niveau de contrainte moyenne des cycles, et l'histoire des contraintes avec notamment l'état maximal de contraintes et le cisaillement initial.

Il faut noter que la rupture est généralement contrôlée par l'angle de frottement intergranulaire $\varnothing^{\prime}$, sauf dans le cas des sables très lâches pour lesquels on observe un effondrement brutal de la structure pour des niveaux de contraintes sensiblement inférieurs au niveau correspondant à la courbe intrinsèque.

Dans le cas des sables, l'accumulation des surpressions interstitielles peut conduire à l'annulation cyclique de la contrainte moyenne effective, entraînant de très grandes déformations et une perte totale momentanée de la résistance, particulièrement pour les faibles densités relatives. Ce phénomène constitue la liquéfaction sous chargement cyclique qui, dans les projets, représente une phase ultime contre laquelle il convient de se prémunir et dont certains aspects sont étudiés ci-après.

\subsubsection{La liquéfaction sous chargement cyclique}

Ce phénomène a fait l'objet de nombreuses études et de plusieurs états des connaissances parmi lesquels il convient de citer ceux de SEED (1979b) et de FINN (1981). C'est la raison pour laquelle nous nous limiterons ici aux aspects particuliers de la liquéfaction pouvant intéresser les pentes et les ouvrages de soutènement.

\section{INVERSION DU SENS DU CISAILLEMENT}

La liquéfaction cyclique ne se produit que dans un chargement où le sens du cisaillement s'inverse à chaque cycle. Comme la figure 7 le montre, une faible inversion suffit pour aboutir à la liquéfaction. Mais 
contrairement à ce qu'ont affirmé LEE et SEED (1967), VAID et FINN (1979) montrent qu'un certain taux de cisaillement inverse est nécessaire. MOKHAM (1983) a retrouvé et quantifié ce résultat.

\section{INFLUENCE D'UNE CONTRAINTE DE CISAILLEMENT INITIALE}

Pour la stabilité aux séismes des pentes sableuses sous l'eau, il est fondamental de savoir si la présence d'une contrainte de cisaillement initiale sur le plan potentiel de glissement a un effet bénéfique ou non sur le potentiel de liquéfaction. La réponse à cette question est évidente d'après le paragraphe précédent : tout cisaillement initial augmente la résistance à la liquéfaction.
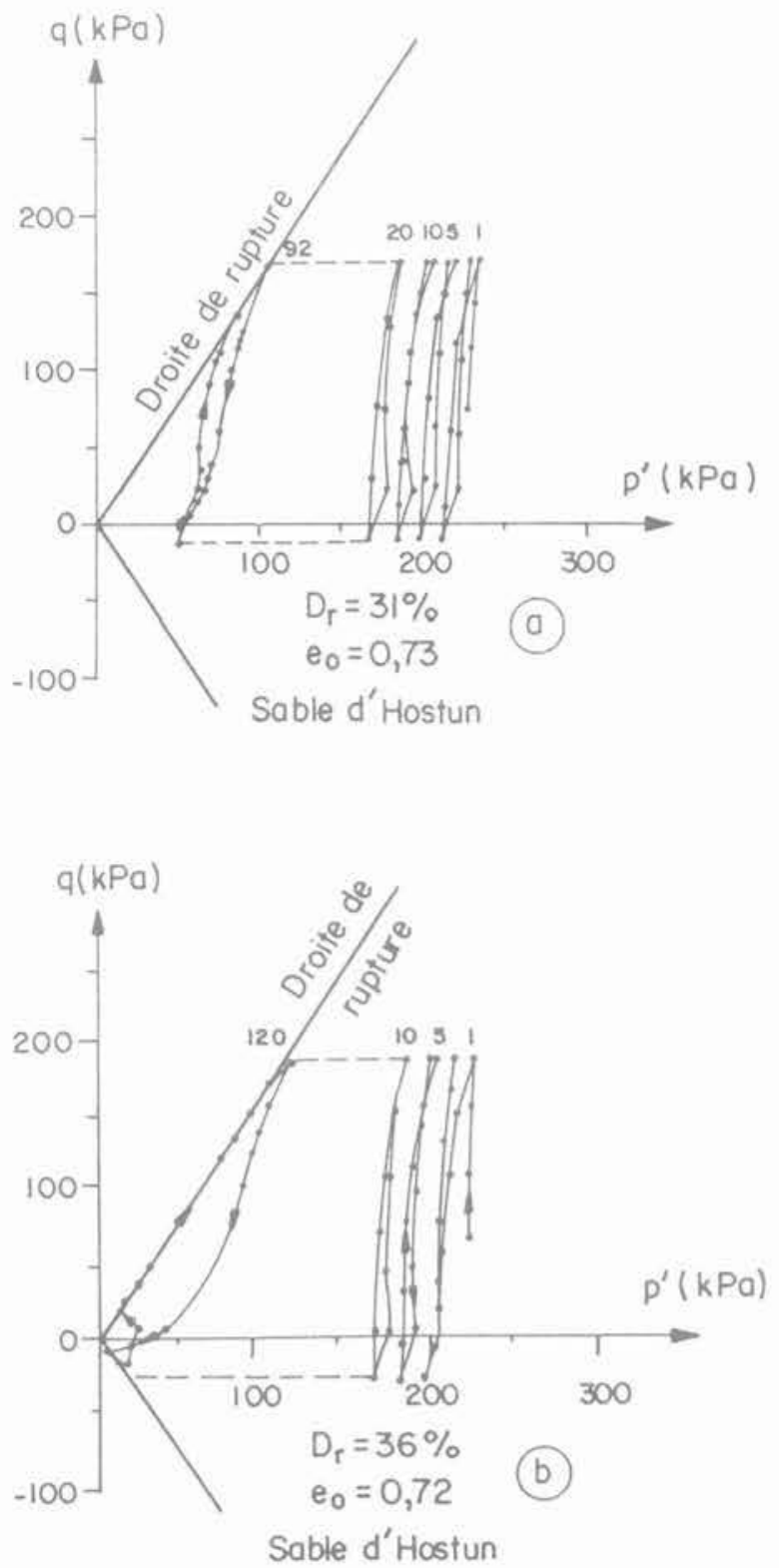

Fig. 7. - Effet de l'amplitude de l'inversion du cisaillement lors des cycles sur la liquéfaction d'un sable (MOKHAM, 1983).
Cependant, si l'on s'intéresse à la résistance au cisaillement cyclique telle que nous l'avons définie en 2.1., VAID et FINN (1979), ainsi que VAID et CHERN (1983), ont montré que celle-ci pouvait augmenter, diminuer ou rester inchangée selon la valeur de la contrainte de cisaillement initiale, de la densité relative du sable et de la valeur de la déformation retenue pour caractériser la rupture. La figure 8 met en évidence le rôle de ces divers paramètres. Globalement on peut dire que, pour les faibles densités relatives, une forte contrainte de cisaillement initiale diminue la résistance au cisaillement cyclique.

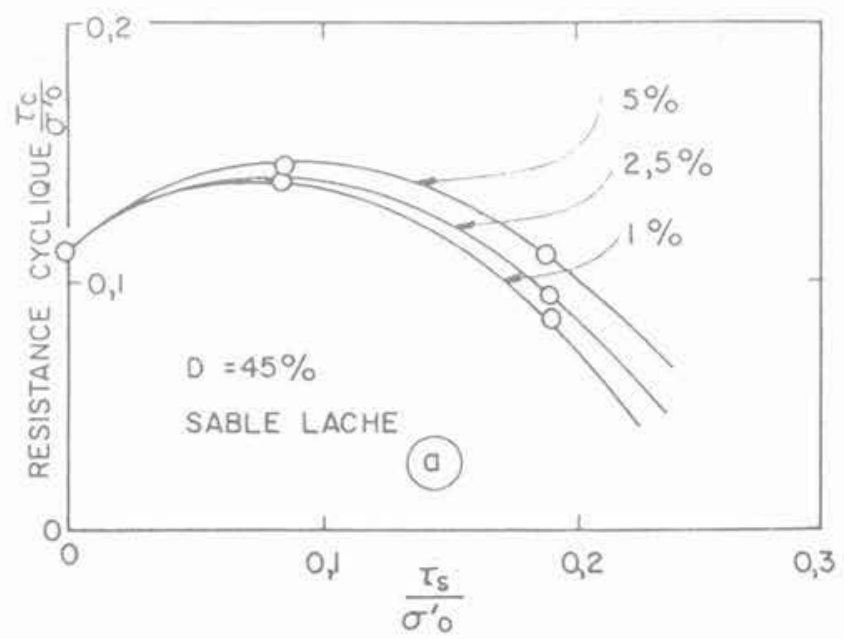

(a) Sable lâche

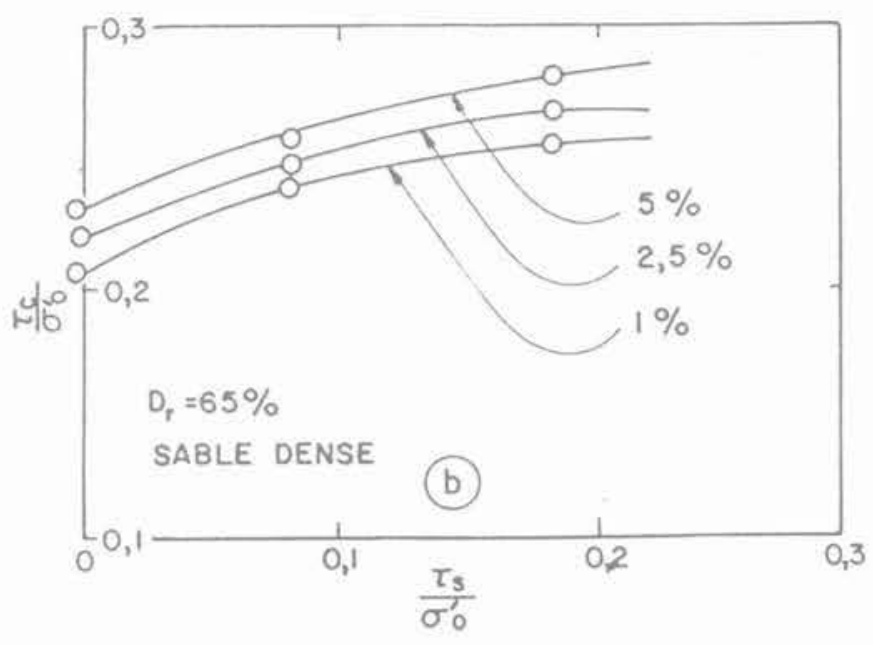

(b) Sable dense

Fig. 8. - Effet d'une contrainte de cisaillement initiale $\tau_{0}$ sur la résistance au cisaillement cyclique définie pour différentes valeurs de la déformation obtenue en dix cycles (VAID et CHERN, 1983).

Comme nous le verrons plus loin, le cas des sables effondrables, donc très lâches, met en jeu des phénomènes spécifiques et l'influence de la contrainte de cisaillement initiale y est très défavorable. 


\section{INFLUENCE DE LA CONTRAINTE MOYENNE}

La contrainte moyenne $\sigma_{\text {mo }}^{\prime}=\sigma_{\text {vo }}^{\prime}(1+2 \mathrm{Ko}) / 3$ agit sur la résistance à la liquéfaction cyclique comme le montre la figure 9 (BHATIA, 1980). Pour un nombre donné de cycles, ces deux grandeurs sont proportionnelles.

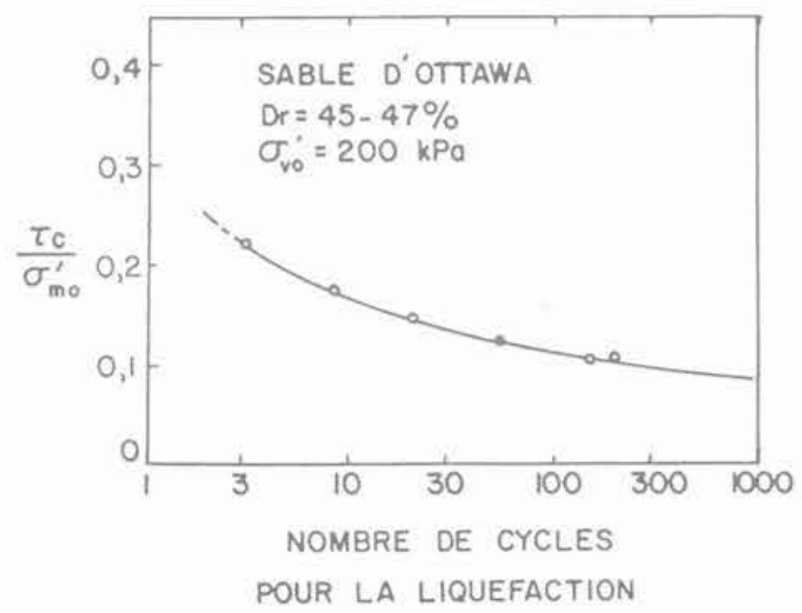

Fig. 9. - Résistance à la liquéfaction exprimée par rapport à la contrainte moyenne (BATHIA, 1980).

\section{INFLUENCE DU DEGRÉ DE SATURATION}

MARTIN et al. (1978) ont fait une étude théorique de l'influence du degré de saturation sur la résistance à la liquéfaction. Leur analyse repose, d'une part sur une formule de prévision de la génération de surpressions interstitielles, d'autre part sur la formule de Koning (1963) donnant le module de déformation volumique du mélange eau-air dans un sol non saturé. La figure 10 montre qu'une baisse de $2 \%$ du degré de saturation, à partir de la saturation complète, multiplie par 3 environ la résistance à la liquéfaction. Ce point doit être pris en considération à la fois pour les projets et pour les essais effectués en laboratoire, mais il nécessite d'être confirmé expérimentalement.

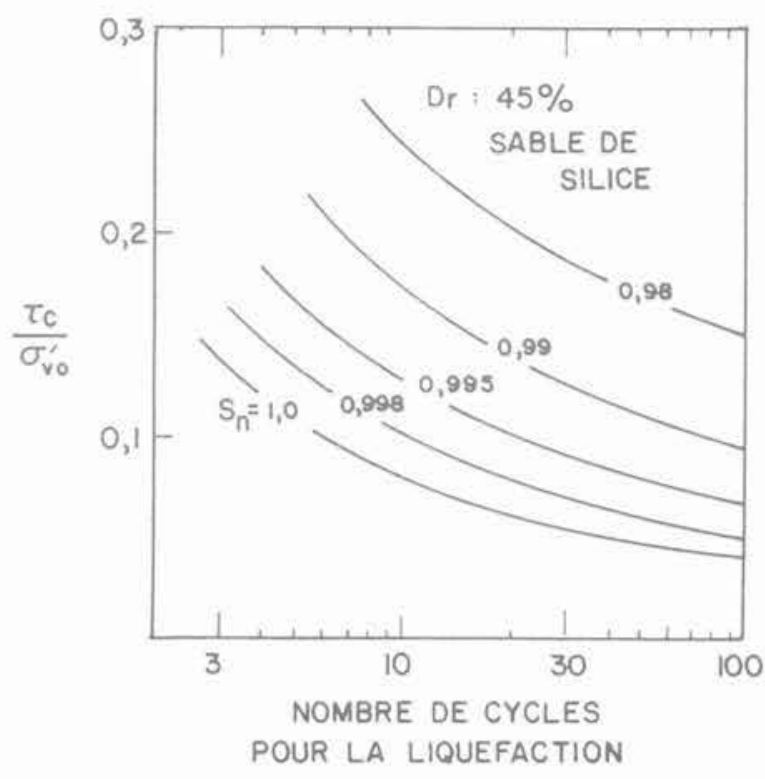

Fig. 10. - Influence du degré de saturation sur le potentiel de liquéfaction (MARTIN et al., 1978).

\subsubsection{Le cas particulier des sables effondrables}

Dans certains sables, dont la densité relative est inférieure à $45 \%$ et la structure instable, VAID et CHERN (1983) ont observé une augmentation brutale de la déformation et de la pression interstitielle, s'amorçant dans le domaine contractant et ne cessant que lorsque le chemin de contraintes est rentré dans le domaine dilatant. Ce phénomène se produit même en l'absence

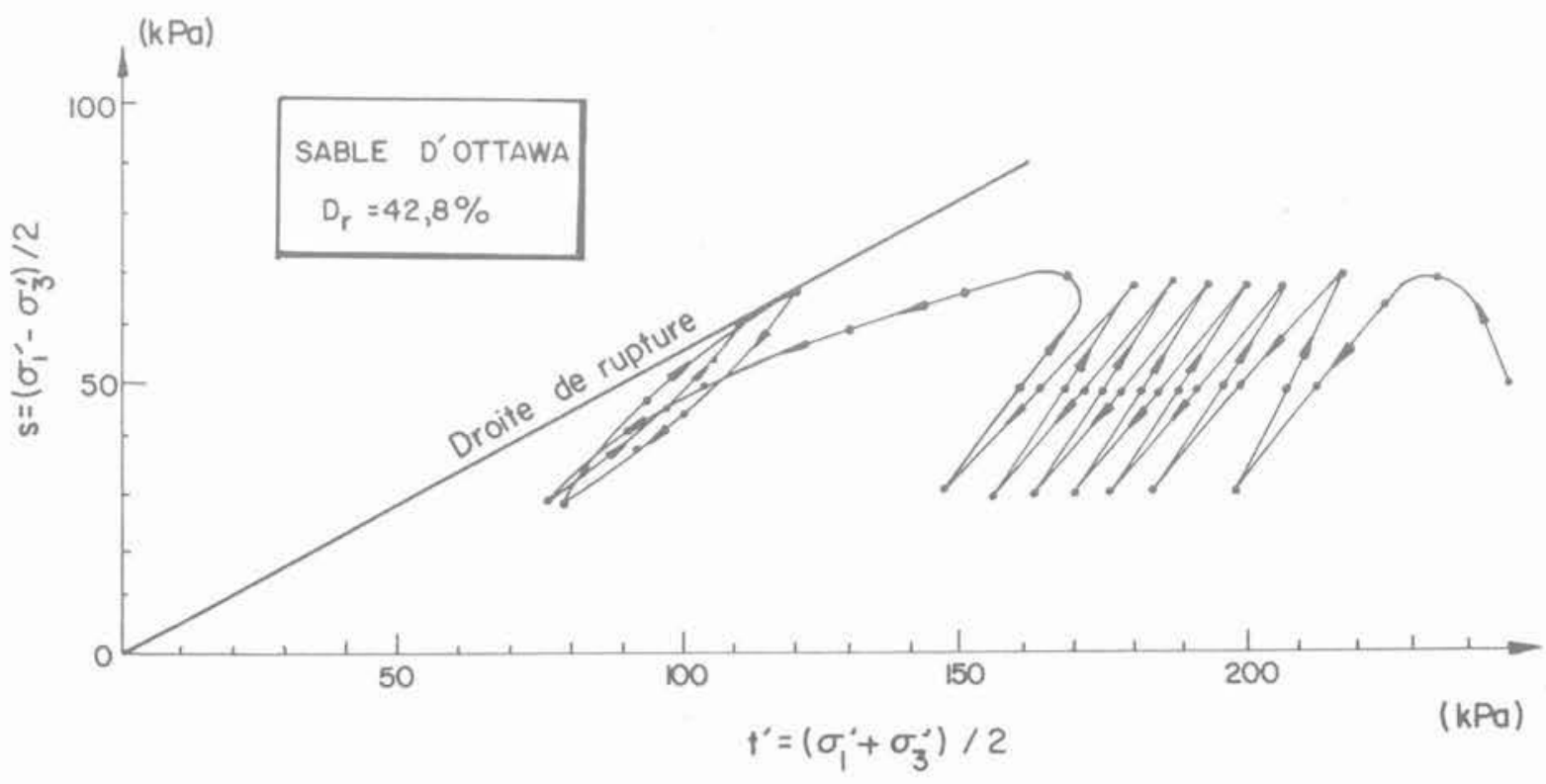

Fig. 11. - Phénomène de déformation d'écoulement (VAID et CHERN, 1983). 
de liquéfaction cyclique, comme cela est présenté à la figure 11. Ces auteurs ont montré la similitude de ce comportement, qu'ils qualifient de déformation d'écoulement, avec la liquéfaction statique sous chargement monotone telle qu'elle a été étudiée par G. CASTRO (1975).

Récemment SLADEN et al. (1985) ont montré qu'il s'agissait bien du même phénomène, caractéristique des sables effondrables, et que celui-ci s'initie de façon systématique lorsque l'état du sable atteint, dans l'espace (p, q, e), une surface particulière appelée surface d'effondrement. Il s'agit d'un cylindre dont la génératrice est la ligne d'état critique et dont la direction est une droite du plan $e=0$. La figure 12 montre, dans le plan (p, q), la position de la droite d'effondrement : elle passe par le point d'état critique sur la ligne de rupture. Tous les états de contraintes, situés entre la droite d'effondrement et la droite de rupture, sont instables, c'est-à-dire qu'en condition non drainée ils évoluent, à la suite de l'effondrement, vers le point d'état critique.

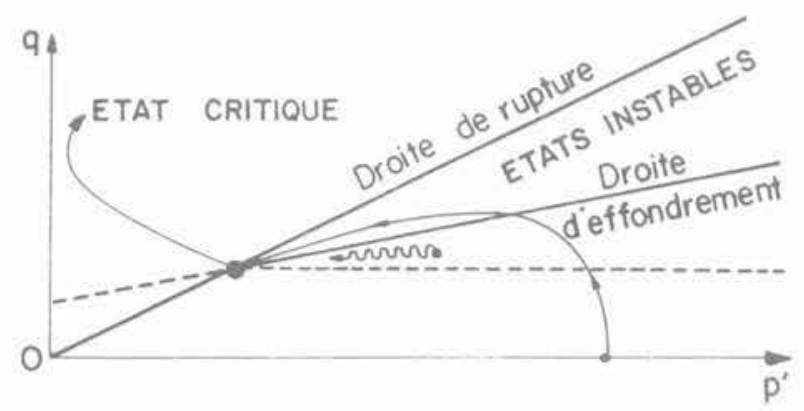

Fig. 12. - Théorie de la surface d'effondrement pour les sables effondrables (SLADEN, 1985).

Cette particularité des sables effondrables conduit, entre autres, à deux différences notables dans le comportement, qui sont détaillées ci-après.

Contrairement au cas des sables de densité relative forte ou moyenne, l'existence d'une contrainte de cisaillement initiale peut diminuer considérablement la résistance au cisaillement cyclique non drainé d'un sable effondrable et entraîner un processus de déformation d'écoulement conduisant à de très larges déformations. VAID et CHERN (1983) ainsi que SLADEN et al. (1985) ont attribué ce phénomène au fait que l'état initial du matériau se trouve rapproché de la surface d'effondrement. Ainsi un chargement cyclique de très faible amplitude peut être suffisant pour provoquer une perte de résistance considérable en peu de cycles si la contrainte de cisaillement initiale est importante et cela même sans inversion du sens du cisaillement. $\mathrm{Ce}$ phénomène explique les glissements spontanés observés dans les pentes marines des grands dépôts sableux comme ceux de la côte de la mer du Nord (KROEZEN et al., 1982).

SLADEN et al. (1985) ont montré, qu'à indice des vides constant, un pourcentage de fines de $12 \%$ augmentait sensiblement la susceptibilité au phénomène d'effondrement et considérablement la perte de résistance du sable après effondrement. Ce phénomène est dû au fait que la présence de fines, bien que ne changeant pratiquement pas l'angle de frottement interne $\varnothing^{\prime}$ du sable, abaisse de manière importante la surface d'effondrement dans l'espace (p, q,e). Ce point mérite d'être noté dans la mesure où l'influence des fines agit ici en sens opposé de ce qui a été observé pour la liquéfaction cyclique des sables non effondrables.

\section{PENTES ET TALUS}

\subsection{Phénomènes et mécanismes dans une pente sous séisme}

Une accélération horizontale cyclique sur une pente a pour effet de provoquer des efforts d'inertie horizontaux et des déformations dans la masse, ces dernières s'accumulant à chaque cycle. La distribution de ces déformations peut être très variable mais schématiquement les déplacements se concentrent près de la surface dans le cas d'un matériau granulaire non saturé et se situent plus en profondeur dans le massif, dans le cas de sols cohérents. Compte tenu du caractère transitoire de la sollicitation, ces déplacements peuvent conduire à des désordres sans pour autant qu'il y ait rupture. En particulier, la pente après séisme peut se retrouver dans un état parfaitement stable. Le niveau des déplacements ou des déformations, susceptibles d'être provoqués par un séisme, constitue ainsi un critère de stabilité qu'il est intéressant de pouvoir quantifier.

Si les sols rencontrés sont saturés, la sollicitation sismique créée des surpressions interstitielles qui ont pour conséquence d'augmenter les déformations et de diminuer la résistance au cisaillement. Dans certains cas, la perte de résistance au cisaillement peut être suffisamment grande pour entraîner une rupture statique après le séisme comme cela a été observé (SEED, 1981).

Dans le cas de pentes sableuses immergées, il peut se produire une liquéfaction sous l'accumulation des pressions interstitielles, ce qui provoque généralement des désordres considérables.

La stabilité des pentes sous séisme a principalement été étudiée à l'occasion des barrages et il faut constater que peu d'observations ont été faites à propos des désordres survenus dans les pentes naturelles.

Ce chapitre sera consacré à l'examen des différentes méthodes de dimensionnement et d'étude de la stabilité des pentes sous séismes. Elles seront classées en trois grandes catégories :

- les méthodes pseudostatiques;

- la méthode de NEWMARK et les méthodes dérivées;

- la méthode de SEED et les méthodes dérivées.

Il convient de remarquer que toutes ces méthodes sont en contraintes totales. La prévision des surpressions interstitielles provoquées dans un massif de sol saturé par un séisme reste en effet délicate et difficile, même si plusieurs méthodes ont été proposées (MARTIN et al., 1975; ISHIHARA et al., 1975). En effet, comme l'a 
précisé WHITMAN (1979), la prévision des surpressions interstitielles nécessite plusieurs étapes qui sont d'ailleurs couplées les unes aux autres :

$1^{\circ}$ L'analyse de la rêponse dynamique du massif de sol pour déterminer les contraintes et/ou les déformations provoquées par le séisme.

$2^{\circ}$ La détermination de la vitesse de génération des surpressions interstitielles, en supposant qu'il n'y ait aucun mouvement de l'eau interstitielle.

$3^{\circ}$ L'étude de la diffusion et de la dissipation des surpressions interstitielles ainsi générées.

Pour les mêmes raisons, les modèles de comportement dynamique en contraintes effectives, qui ont été développés (FINN et al., 1976; GHABOUSSI et DIKMAN, 1978; ZIENKIEWICZ, 1978; ISHIHARA, 1980), ne sont guère utilisables dans les calculs aux éléments finis.

\subsection{Les méthodes pseudostatiques de calcul à la rupture}

Ce type de méthode est dérivé de la méthode classique d'analyse de la stabilité statique d'une pente en rupture circulaire. On considère en effet, qu'aux forces volumiques classiques de gravité s'ajoute une force volumique $\gamma \overrightarrow{\mathrm{k}}$ d'intensité constante, destinée à simuler l'effet des forces d'inertie dues au séisme $(\gamma$ : poids volumique du matériau). Dans cette approche, le séisme est donc entièrement caractérisé par la donnée du vecteur: $\overrightarrow{\mathrm{k}}=\overrightarrow{\mathrm{a}} / \mathrm{g}$ où $\overrightarrow{\mathrm{a}}$ désigne la densité volumique d'accélération d'inertie. Cette méthode a été introduite par TERZAGHI dès 1950; elle fut et demeure encore largement utilisée compte tenu des nombreux développements qui ont été faits en statique (méthode de BISHOP, méthode des perturbations, méthode en rupture non circulaire, ...).

Le vecteur sismique $\vec{k}$ a deux composantes : le coefficient sismique horizontal $\mathrm{k}_{\mathrm{h}}$ dont la valeur est prépondérante, le coefficient sismique vertical $\mathrm{k}_{\mathrm{y}}$ souvent négligé. Les valeurs couramment utilisées pour $k_{h}$ vont de 0,05 à 0,15 aux États-Unis et de 0,15 à 0,25 au Japon. Ce choix reste essentiellement empirique, sans être réellement calé sur l'expérience et sur les observations (SEED, 1966a, 1966b, 1969). Le seul moyen de connaître les valeurs des coefficients sismiques réellement à prendre en compte consiste à analyser la stabilité de barrages qui se sont rompus au cours de séismes. SEED (1969) a ainsi montré que pour expliquer la rupture en 1925 du barrage de Sheffield en Californie, pour lequel l'accélération maximale de la base était de $0,15 \mathrm{~g}$, une valeur de $k_{\mathrm{b}}$ comprise entre 0,1 et 0,17 était suffisante. Cependant, il a calculé que pour des accélérations du sol supérieures, de l'ordre de $0,4 \mathrm{~g}$ à $0,5 \mathrm{~g}$, c'est-à-dire typiques des tremblements de terre californiens, un coefficient sismique de 0,3 au minimum était nécessaire. Avec une telle imprécision sur la valeur de $\overrightarrow{\mathrm{k}}$ à prendre en compte, la notion même de coefficient de sécurité perd sa signification puisque l'on pourrait avoir des ruptures pour des coefficients de sécurité supérieurs à 1 .
On peut bien sûr adopter un point de vue très conservatif en prenant pour $\mathrm{k}_{\mathrm{h}}$ la valeur maximale de l'accélération horizontale dans la pente. SEED (1966) a fait ce calcul par deux méthodes différentes, pour un barrage de $100 \mathrm{~m}$ de haut, soumis au tremblement de terre d'El Centro (1940), dont l'accélération maximale à la base était de $0,3 \mathrm{~g}$. Le résultat est présenté à la figure 13 . II montre une grande divergence entre les valeurs classiquement utilisées et les valeurs obtenues par le calcul, ce qui paraît normal puisque l'on a remplacé des accélérations transitoires par des accélérations permanentes prises égales aux accélérations maximales.

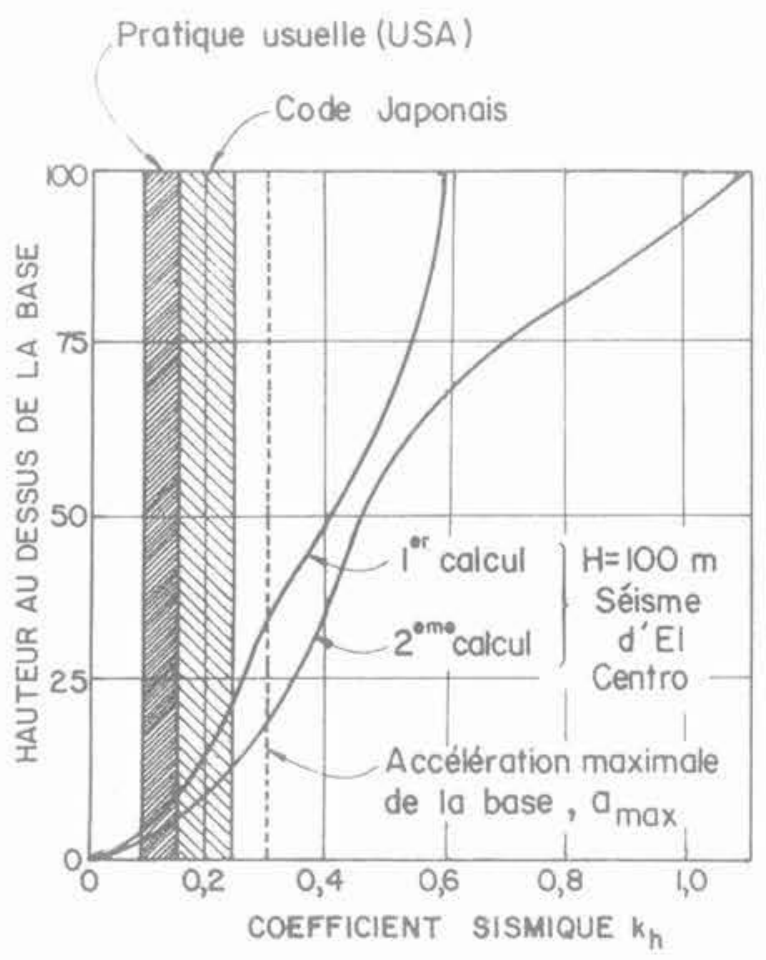

Fig. 13. - Valeurs usuelles et calculées du coefficient sismique.

Dans le domaine des pentes naturelles, TANIGUCHI et SASAKI (1985), qui ont analysé un glissement de terrain de $270000 \mathrm{~m}^{3}$ provoqué par le séisme de Naganokon Seibu en 1984 au Japon, ont montré que le coefficient sismique semblait convenablement donné par l'une ou l'autre des deux formules suivantes :

$$
\begin{aligned}
& \mathrm{k}=0,65 \frac{\mathrm{a}_{\max }}{\mathrm{g}} \\
& \mathrm{k}=\frac{1}{3}\left(\frac{\mathrm{a}_{\max }}{\mathrm{g}}\right)^{1 / 3}
\end{aligned}
$$

En tout état de cause, l'impossibilité d'évaluer précisément $k$ en fonction d'un séisme défini constitue le défaut majeur de la méthode pseudostatique. 


\subsection{La méthode aux déplacements de NEWMARK (1965)}

NEWMARK a proposé en 1965 une méthode de calcul des déplacements d'une pente soumise à un séisme. La méthode s'applique exclusivement à un sol granulaire sec et homogène. Les déplacements sont supposés se produire parallèlement à la pente selon une cinématique de glissement plan. Le comportement du sol est supposé rigide-plastique, les déplacements ne se produisant que le long de la surface de rupture.

Au cours des cycles, il y a alternativement glissement relatif du sol en surface vers l'aval et entrainement de cette partie dans le mouvement général d'oscillation. Il en résulte par saccades une reptation d'une couche superficielle du sol vers le bas de la pente.

De manière simplifiée, telle que l'a présentée NEWMARK, on peut considérer un accélérogramme en créneaux de temps élémentaire $t_{0}$. Pour une accélération horizontale orientée vers l'amont, il y a glissement lorsque l'accélération A.g est supérieure à une valeur limite $A^{\prime}, g$, fonction de l'angle $\theta$ de la pente et de l'angle de frottement interne $\varnothing$ :

$$
\mathrm{A}^{\prime}=(\cos \theta \cdot \operatorname{tg} \phi+\sin \theta) /(\sin \theta \cdot \operatorname{tg} \phi-\cos \theta)
$$

Le déplacement relatif $\delta$, à chaque cycle, est obtenu par une double intégration :

$$
\delta=\frac{V^{2}}{2 g A^{\prime}}\left(1-\frac{A^{\prime}}{A}\right)
$$

où $\mathrm{V}=\mathrm{Agt}_{0}$.

La méthode a été vérifiée sur des essais en modèles réduits à la table vibrante qui ont donné des résultats assez satisfaisants (NEWMARK, 1965).

Cette méthode a ensuite été améliorée par GOODMAN et SEED (1966) qui procèdent numériquement à une double intégration sur l'accélérogramme, pour évaluer le déplacement cumulé, et qui tiennent compte d'une éventuelle diminution de la résistance au cisaillement par radoucissement d'un cycle à l'autre, comme indiqué à la figure 14. Ayant réalisé de nombreux essais en modèles réduits, ils font remarquer que la méthode est très sensible à la valeur de l'angle de frottement interne du sol.

MAKDISI et SEED (1978) ont repris la méthode de GOODMAN et SEED (1966) en tenant compte d'une cinématique de rupture circulaire ce qui est une première amélioration sensible par rapport à la méthode de NEWMARK, qui est limitée aux glissements plans parallèles à la pente. En outre, l'accélération utilisée tient compte de la réponse du massif vis-ầ-vis de l'accélération excitatrice du sol. Les résultats de la méthode sont présentés sous forme d'abaques permettant d'évaluer le déplacement de la zone en glissement en fonction de la magnitude du séisme, du rapport $\frac{A^{\prime}}{A}$ de l'accélération limite $A^{\prime}$ g à l'accélération maximale de réponse du massif et de la première période propre $T$. des vibrations du massif.
La méthode a été testée sur un calcul a posteriori effectué sur le barrage de Chabot, en Californie, qui fut soumis au tremblement de terre de 1906 (MAKDISI et SEED, 1978). Récemment YZIQUEL et al. (1981) ont appliqué cette méthode au barrage d'Ait Chouarit $(\mathrm{H}=140 \mathrm{~m})$ au Maroc. Le déplacement horizontal maximal obtenu a été de $0,82 \mathrm{~m}$ pour un cercle de glissement superficiel en tête du talus amont. L'accélération maximale en crête et sur le parement amont était de $0,54 \mathrm{~g}$.

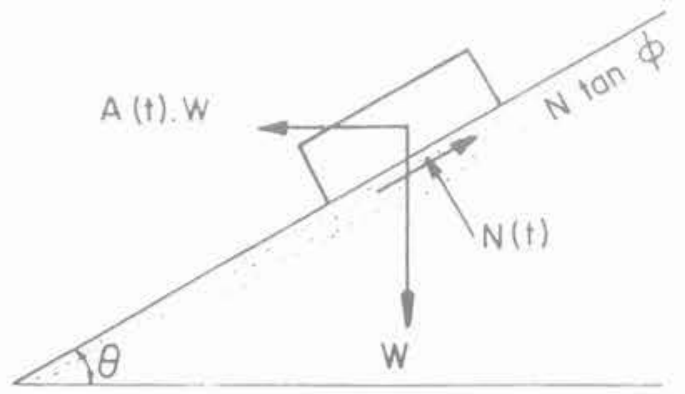

(a) Equilibre du bloc ou cours du temps

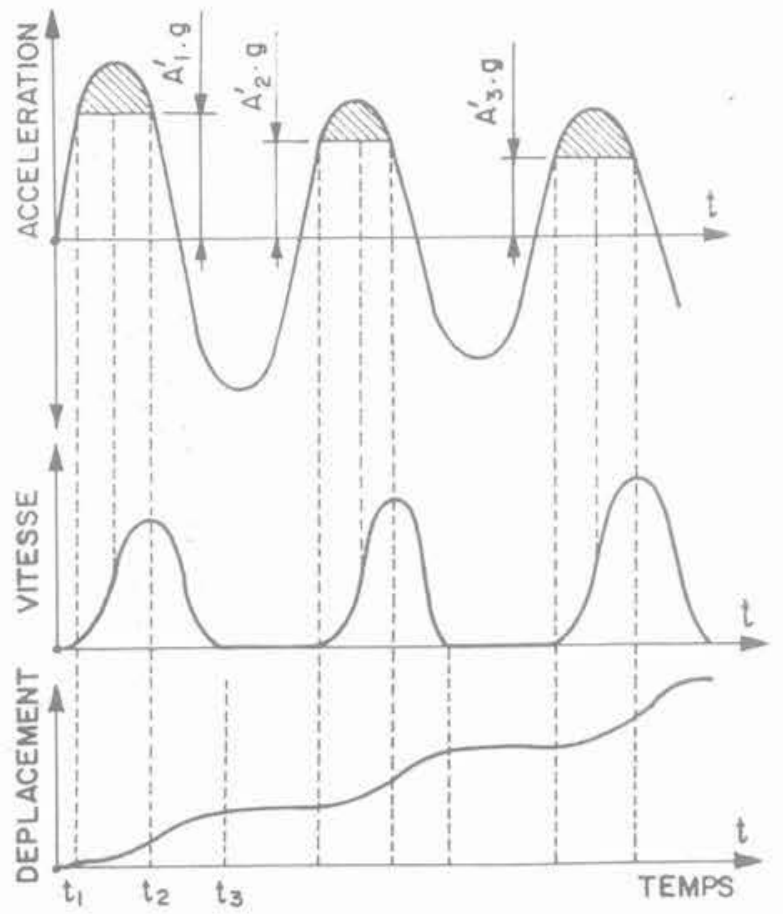

(b) Double intégration de l'accélérogramme

Fig. 14. - Méthode aux déplacements pour la stabilité dynamique des pentes (NEWMARK, 1965). 


\subsection{La méthode de SEED et les méthodes dérivées}

\subsubsection{Méthode du chemin de contraintes (SEED, 1966)}

Les méthodes pseudostatique et de NEWMARK et celles qui en dérivent font appel à des paramètres de résistance au cisaillement supposés peu variables au cours d'une sollicitation cyclique. Elles sont donc inadaptées au cas des sols saturés en sollicitation cyclique non drainée, sables ou argiles, dans lesquels un déviateur cyclique provoque une accumulation de surpressions interstitielles responsables d'une détérioration notable des caractéristiques de résistance et de déformation à court terme.

SEED (1966) a proposé une méthode de calcul de la stabilité d'un remblai, constitué d'un sol saturé homogène, tenant compte des propriétés particulières de la réponse d'un tel matériau à un chargement dynamique et permettant d'évaluer les déformations et les déplacements du massif sans faire d'hypothèse restrictive sur la cinématique de la rupture. Cette approche a fait depuis 1966 l'objet de divers perfectionnements provenant principalement d'un recours accru aux techniques numériques (éléments finis) et des progrès réalisés en laboratoire dans la connaissance du comportement dynamique des sols saturés. Cependant, la démarche fondamentale reste inchangée.

La méthode fait partie des méthodes dites du « chemin de contraintes ", consistant à calculer numériquement les contraintes dans un massif à l'aide d'une loi de comportement appropriée, puis à utiliser ces résultats dans un programme d'essais en laboratoire sur des échantillons représentatifs pour en déduire le champ des déformations.

La succession des opérations est ainsi la suivante :

$1^{\circ}$ En premier lieu, un calcul, généralement en élasticité non linéaire, fournit les contraintes existant dans le remblai avant séisme, contraintes qui jouent, comme on l'a vu en 2.3.2., un rôle essentiel dans la résistance au cisaillement cyclique non drainée. Les contraintes cycliques apportées par le séisme sont ensuite évaluées en utilisant une loi de comportement viscoélastique linéaire équivalente du matériau. On en déduit en chaque point le nombre $\mathrm{N}$ de cycles sinusoïdaux simulant l'histoire des contraintes cycliques calculées.

$2^{\circ}$ Par des essais cycliques non drainés sur des échantillons en laboratoire, on obtient la famille de droites du plan de MOHR, d'équation :

$$
\tau_{r}=\mathrm{f}\left(\sigma_{0}^{\prime}, \mathrm{N}, \varepsilon_{r}, \tau_{0} / \sigma_{0}^{\prime}\right)
$$

introduite en 2.1. et caractérisant le comportement dynamique du sol.

$3^{\circ} \mathrm{La}$ combinaison des résultats de $1^{\circ}$ et de $2^{\circ}$ permet alors d'obtenir en tout point les valeurs des déformations principales (notamment $\mathcal{E}_{1}$ ), appelées déformations potentielles car elles ne sont qu'une approximation de la réalité.
La connaissance complète des champs de déformations et de déplacements nécessite des hypothèses complémentaires, à la fois sur la direction des déformations principales et sur l'intégration des déformations (conditions de compatibilité) (LEE, 1974; SERFF et al., 1976).

La stabilité de la pente pendant et après le séisme peut être quantifiée par un coefficient de sécurité dans un calcul classique en rupture circulaire (généralement méthode des tranches), en utilisant les relations précédentes donnant la résistance au cisaillement cyclique non drainée $\tau_{r}$. On y fixe arbitrairement la valeur de $\varepsilon_{z}$, sachant qu'un coefficient de sécurité égal à 1 signifie que, le long de la surface de rupture potentielle, la déformation moyenne est alors égale à $\varepsilon_{x}$. L'interprétation d'un tel coefficient de sécurité reste malgré tout délicate, car le lien avec les déplacements réels du massif est très difficile à établir.

Cette méthode a été testée sur huit barrages ayant subi un séisme (SEED, 1979a). Elle a permis de retrouver a posteriori la bonne tenue ou la rupture des ouvrages étudiés, ainsi que les traits généraux de la cinématique observée. Des études particulièrement détaillées ont été publiées pour les cas du barrage de Sheffield (SEED et al., 1969) et des barrages de San Fernando (SEED et al, 1975). De plus, elle a été largement utilisée pour la prévision du comportement de remblais vis-à-vis de séismes de projet, comme l'indiquent les nombreuses références citées par SEED (1979a).

La figure 15 présente les résultats de la méthode dans le cas du barrage Hawkins $(\mathrm{H} \simeq 20 \mathrm{~m})$ en Californie (LEE et ROTH, 1977) : (a) lignes de la déformation potentielle $\varepsilon_{1}$, (b) surfaces de rupture circulaire potentielle et coefficients de sécurité correspondants, (c) déplacements du barrage. Les calculs ont été effectués pour le séisme probable le plus sévère, à savoir : $M=8,25$ et $\mathrm{a}_{\max }=0,42 \mathrm{~g}$. La rupture du matériau de remblai a été définie pour $\varepsilon_{r}=5 \%$. Il est intéressant de constater que pour une telle valeur de $\varepsilon_{x}$, un coefficient de sécurité de 1,2 correspond à un déplacement maximal de $11,5 \mathrm{~cm}$ en crête.

\subsubsection{Méthode de seuil (SEED, 1969; LEE, 1974)}

A côté de la méthode du chemin de contraintes telle qu'elle a été exposée précédemment, SEED et al. (1969) ainsi que LEE (1974) ont proposé une méthode de seuil consistant à déterminer les zones d'un barrage où la contrainte globale (statique + dynamique) de cisaillement $\tau$ est supérieure à la contrainte de seuil $\tau_{\ell}$.

Une telle méthode a été appliquée par LEE (1974) pour déterminer les domaines de déformations irréversibles supérieures à un seuil donné sous l'effet d'un séisme, et dans ce cas, il utilise comme critère de seuil la famille de droites $\tau_{t}=f\left(\sigma_{0}^{\prime}, N, \varepsilon_{t}, \tau_{0} / \sigma_{0}^{\prime}\right)$ déterminée par un programme d'essais de cisaillement cyclique. Suivant la valeur retenue pour la déformation $\mathcal{E}_{\mathrm{r}}$ à la rupture, on distingue des zonages différents du barrage. On trouvera des illustrations de cette méthode dans LEE et al. (1977) ainsi que dans POST et FLORENTIN (1981) qui présentent une analyse du risque 
de déformations irréversibles (seuil fixé à $5 \cdot 10^{-4}$ ) du barrage de Verney $(\mathrm{H}=43 \mathrm{~m})$ dans le cas d'une accélération maximale en crête de $0,45 \mathrm{~g}$.

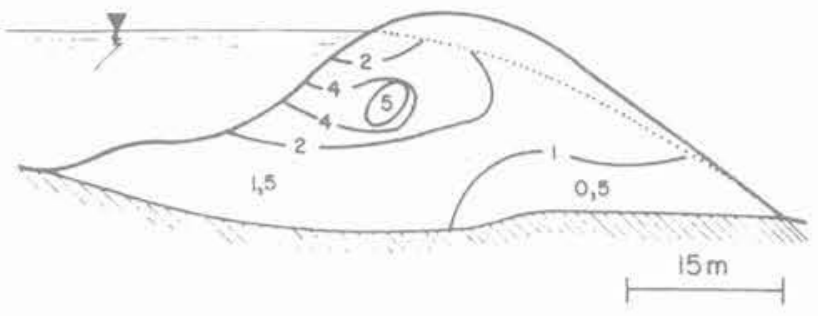

(a) Lignes de déformation (\%) potentielle

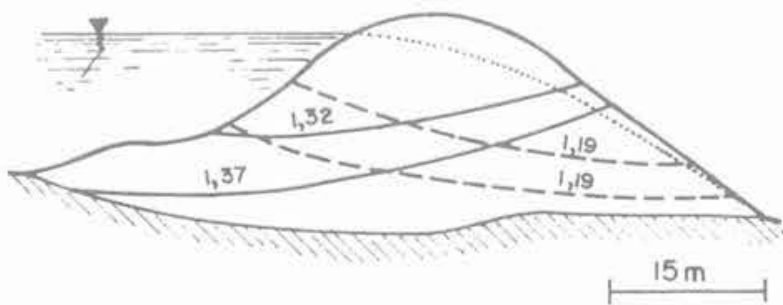

\section{(b) Surfaces de rupture potentielle}

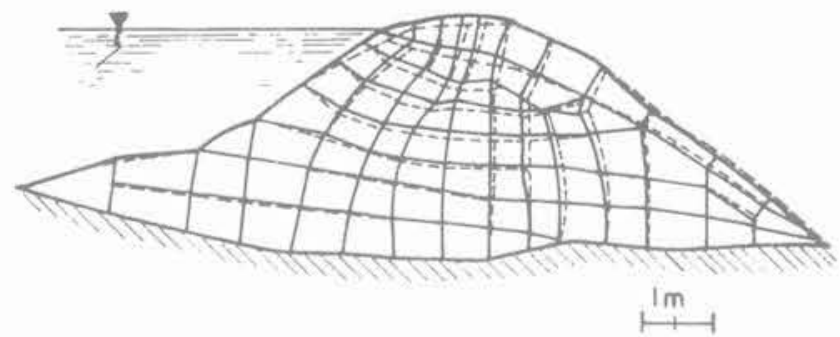

C Déplacements du barrage

Fig. 15. - Méthode de SEED appliquée au barrage de Hawkins (LEE et ROTH, 1977).

SEED applique couramment cette méthode depuis 1969 pour l'étude du risque de liquéfaction des barrages construits avec des matériaux sableux. Dans ce cas, la contrainte de seuil est constituée par la résistance à la liquéfaction. La méthode peut être utilisée à la fois pour les risques de liquéfaction dynamique et statique (cf. la note présentée à ces journées par F. BLONDEAU). Il est préférable de procéder par étapes en scindant la sollicitation dynamique en plusieurs sollicitations successives de faible durée, car cela permet de mieux appréhender la cinématique et la propagation de la liquéfaction. La figure 16 montre le résultat d'une telle analyse dans le cas de la rupture du barrage de Sheffield (SEED et al., 1969).

Les méthodes présentées dans ce paragraphe 3.4. ont à la fois une base expérimentale et une base théorique. Leur intérêt est qu'elles sont en général convenablement calées sur des cas réels, même si ceux-ci ne sont pas très nombreux. Leur inconvénient est qu'elles restent malgré tout approximatives, tant vis-à-vis du comportement dynamique du sol que du développement des déformations au cours du séisme.
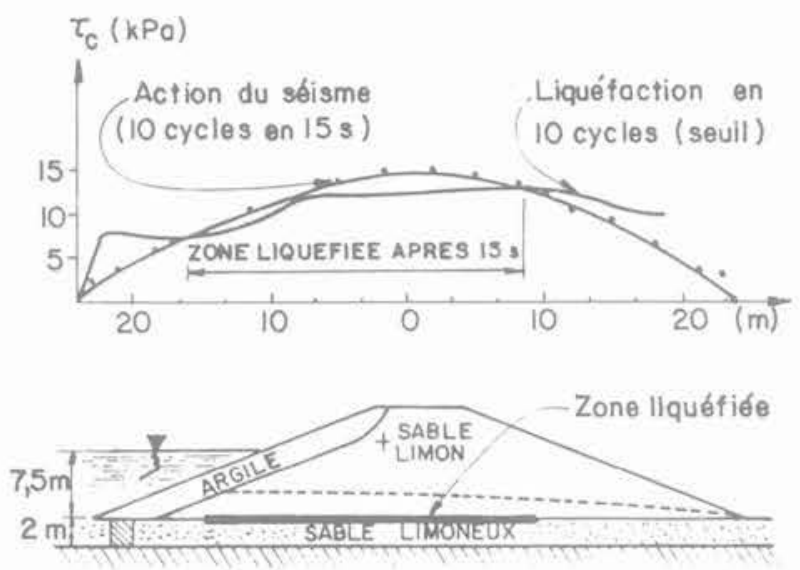

Fig. 16. - Analyse de la liquéfaction dans la rupture du barrage de Sheffield (SEED et al., 1969). Méthode du seuil.

\subsection{Les méthodes aux déformations par utilisation des éléments finis}

La plupart des méthodes précédemment exposées font appel au calcul par éléments finis pour la détermination des contraintes dans les barrages. L'étape ultérieure consisterait à intégrer dans un programme en dynamique une loi de comportement suffisamment représentative sans faire appel à un trop grand nombre de paramètres difficiles à déterminer. Il n'y a encore que peu d'exemples de tels programmes dans la littérature.

\section{MURS ET SOUTÈNEMENTS}

\subsection{Phénomènes et mécanismes pour un mur de soutènement sous séisme}

Parmi les études qui ont mis en évidence les phénomènes et les déformations rencontrés lors d'une sollicitation sismique s'exerçant sur un mur de soutènement, il faut signaler les synthèses faites par PRAKASH (1981) et par AUBRY et CHOUVET (1985). Elles montrent qu'en dehors des murs-poids rigides, peu d'expérimentations ont été faites sur les autres ouvrages de soutènement. Récemment, des recherches ont été entreprises sur le comportement aux séismes des ouvrages en terre armée afin d'en améliorer les méthodes de dimensionnement et d'en mieux comprendre le comportement (BASTICK et SCHLOSSER, 1985).

D'une façon générale l'effet de la sollicitation sismique sur un mur-poids est caractérisé par un déplacement par à-coups, dont les composantes sont une translation horizontale du mur ainsi qu'une rotation autour de la 
base, vers l'extérieur. Il y a toujours un coin de remblai solidaire du mur et il se crée à l'amont du mur une surface de rupture plane, dont l'inclinaison sur l'horizontale est plus faible que lors d'une rupture statique, cette inclinaison diminuant d'ailleurs avec l'importance de la sollicitation sismique (SEED et WHITMAN, 1970; MURPHY, 1960; AUBRY et CHOUVET, 1985). La figure 17 illustre ce comportement dans le cas d'un essai sur modèle réduit (MURPHY, 1960).

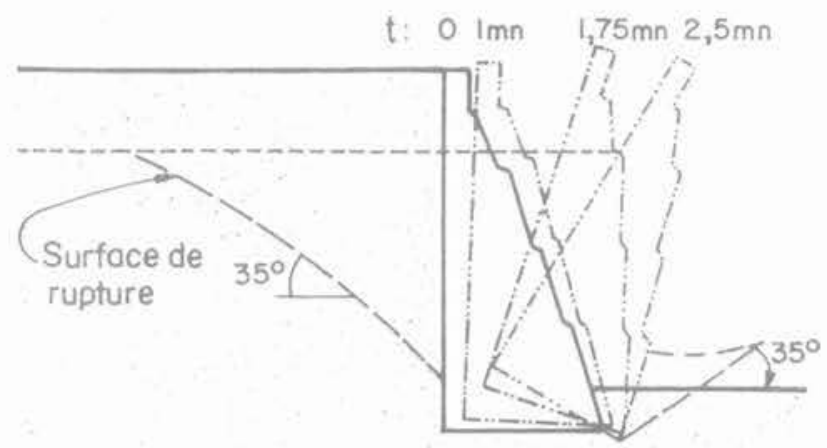

TABLE VIBRANTE $\left(\rightarrow-a_{\text {max }}=0,25 \mathrm{~g}\right)$

Fig. 17. - Cinématique de la rupture d'un modèle réduit de mur-poids dans un essai à la table vibrante (d'après MURPHY, 1960).

Bien que la cinématique de la rupture varie suivant le type d'ouvrages de soutènement, il se dégage le fait que l'inertie de l'ouvrage est un facteur important dans le comportement. Ce point est parfaitement illustré par la figure 18 (AUBRY et CHOUVET, 1985) qui montre le déplacement horizontal de deux murs-poids d'inerties différentes soumis à la même sollicitation dynamique (modèles réduits bidimensionnels). Il avait été omis dans les premières méthodes de dimensionnement proposées dans le rapport général de SEED et WHITMAN (1970), méthodes qui reposaient principalement sur la prise en compte de l'effort de poussée dynamique. Un pas important a été franchi avec l'apport de RICHARDS et ELMS (1979), qui ont proposé, dans l'esprit de la méthode de NEWMARK, de calculer le déplacement de translation du mur sur sa base, faisant de ce fait intervenir l'inertie de celui-ci.

Schématiquement, le mécanisme de la translation horizontale est le suivant, en remarquant que les forces de poussée et de butée dynamique sont respectivement plus forte et plus faible que les forces statiques. Lors d'un cycle, la somme des efforts exercés sur le parement du mur et sur sa base évolue entre deux extrêmes comme l'indique la figure 19 qui montre la variation de cette somme S d'efforts en fonction du déplacement horizontal du mur. L'équation simplifiée du mouvement est alors :

$$
M \cdot \ddot{x}=S(x)-M \cdot \ddot{x}_{e}
$$

où $\mathrm{M}$ représente la masse du mur, $\ddot{x}_{\mathrm{e}}$ l'accélération de la base, et $\ddot{x}$ l'accélération relative. Le mouvement de translation peut, sous de fortes sollicitations, être représenté par une succession de boucles, comme le montre la même figure.

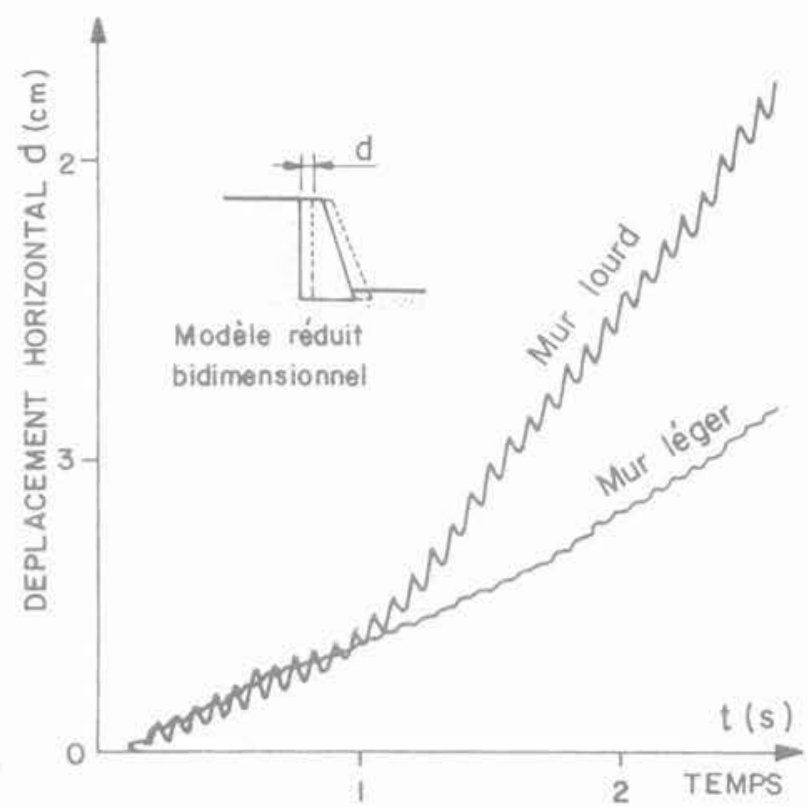

Fig. 18. - Influence de l'inertie sur le déplacement de murs en modèle réduit bidimensionnel à la table vibrante (d'après AUBRY et CHOUVET, 1985).

On peut faire les mêmes remarques en ce qui concerne le phénomène et le mécanisme de rotation du mur autour de sa base. L'inertie joue un rôle important sur l'amplitude de la rotation et il est possible de développer des modèles élastoplastiques pour expliquer le mécanisme.

Le mouvement de translation relatif entre le mur et le remblai a un effet important sur l'amplitude de la pous. sée dynamique. Le mouvement global de rotation influence par contre très directement la distribution des pressions dynamiques et tout particulièrement le point d'application de la résultante.

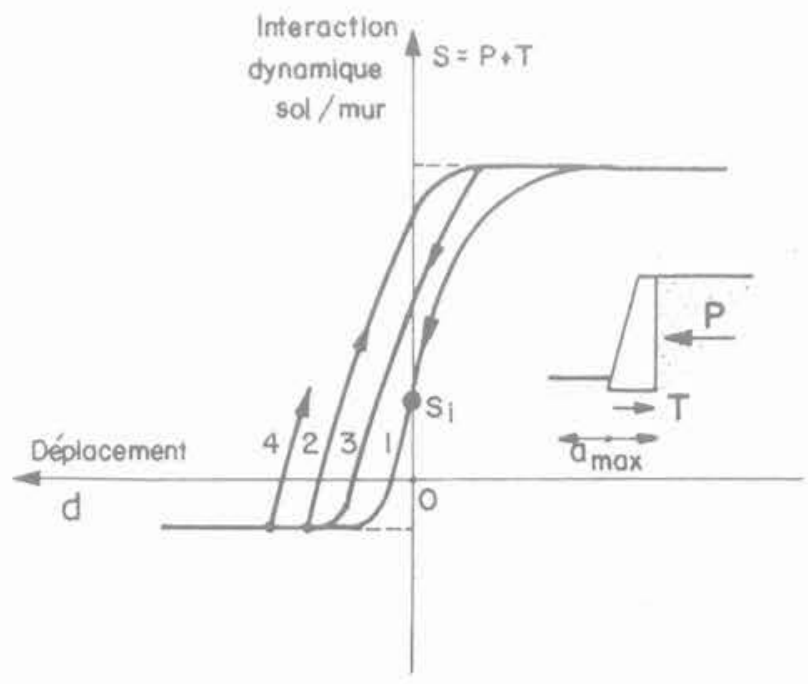

Fig. 19. - Mécanisme de la translation horizontale d'un mur soumis à des vibrations horizontales. 
Dans la pratique la zone influencée par le déplacement d'un mur soumis à l'effet d'un séisme est beaucoup plus grande que celle intéressée par le coin de Coulomb statique, ce qui peut avoir une incidence sur des ouvrages édifiés à proximité. En outre, il faut savoir qu'après un séisme, la poussée statique s'exerçant sur le mur est supérieure à ce qu'elle était auparavant, jusqu'à $30 \%$ d'après NADIM et WHITMAN (1983),

Nous étudierons les deux principales contributions au dimensionnement des murs, d'une part la méthode pseudostatique de MONONOBE-OKABE (1929) pour l'évaluation de la poussée dynamique maximale, d'autre part la méthode de RICHARDS et ELMS (1979) pour l'évaluation des déplacements de translation du mur.

Nous ne traiterons pas le cas où il y a de l'eau derrière le mur, c'est-à-dire le cas où se développent des surpressions interstitielles dans le remblai sous l'effet du séisme. Il n'y a d'ailleurs pratiquement pas de publications à ce sujet, mais l'on peut penser que les méthodes propres à l'étude de la stabilité des pentes saturées sont applicables dans ce cas (par exemple stabilité vis-à-vis de la liquéfaction cyclique).

\subsection{Méthode de MONONOBE-OKABE (1929)}

- La méthode de MONONOBE-OKABE (1929) constitue une extension au cas des murs sous séisme, du calcul de la poussée statique de Coulomb. Elle est limitée à des remblais rigides-plastiques, secs et sans cohésion. MONONOBE et OKABE ont proposé de prendre en compte l'action du séisme par une densité volumique de forces $\gamma \vec{k}$ (identique à celle introduite précédemment dans la méthode pseudostatique en stabilité des pentes). Il s'agissait là des premiers calculs pseudostatiques en mécanique des sols. Cornme dans le calcul classique de Coulomb, on étudie l'équilibre d'un coin en arrière du mur (fig. 20) et on détermine l'effort exercé par le coin sur le mur, c'est-à-dire la poussée dynamique, en minimisant sa valeur par rapport à l'angle $\alpha$ d'inclinaison du coin sur l'horizontale, ce qui donne l'expression :

$$
(\mathrm{Pa})_{\mathrm{dyn}}=\frac{1}{2} \gamma \mathrm{H}^{2}\left(1 \pm \mathrm{k}_{\mathrm{v}}\right) \cdot(\mathrm{Ka})_{\mathrm{dyn}}
$$

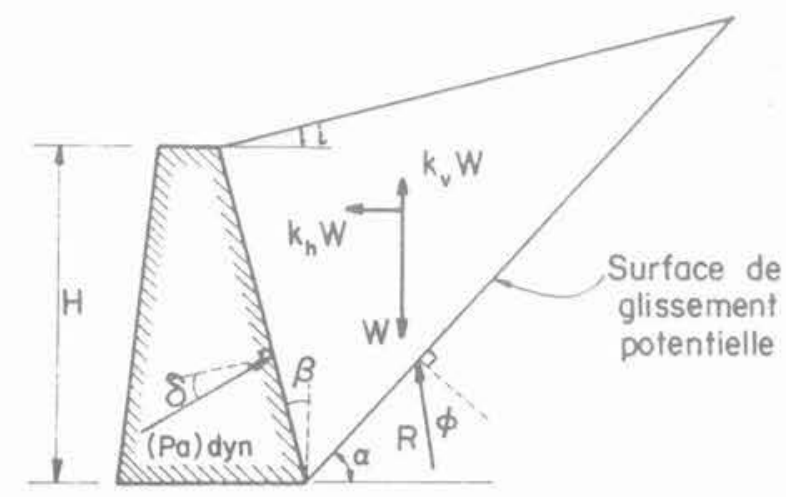

Fig. 20. - Forces prises en compte pour l'équilibre du coin. Méthode pseudostatique de MONONOBE-OKABE (1929). avec :

$$
\text { (Ka) })_{\text {dyn }}=\frac{\cos ^{2}(\Phi-\theta-\beta)}{\cos \theta \cos ^{2} \beta \cos (\delta+\beta+\theta)\left\{1+\left(\frac{\sin (\Phi+\delta) \sin (\phi-\theta-1)}{\cos (\delta+\beta+\theta) \cos (\delta-\beta)}\right)^{\frac{1}{2}}\right\}}
$$

Les notations utilisées dans ces formules sont les suivantes :

$k_{h}$ : accélération horizontale

$k_{v}$ : accélération verticale

$\theta: \operatorname{Arctg}\left(\frac{k_{h}}{1 \pm k_{v}}\right)$

$\gamma$ : poids volumique du sol

$H$ : hauteur du mur

$\Phi$ : angle de frottement interne

$\delta$ : angle de frottement mur/sol

i : angle du remblai derrière le mur

$\beta$ : angle de la face interne du mur avec la verticale

SEED et WHITMAN (1970) ont étudié le rôle de ces divers paramètres sur la valeur calculée de $(\mathrm{Pa})$ dyn . laquelle s'avère fortement croissante en fonction de $\mathrm{k}_{\mathrm{h}}$, i et $\phi$, mais peu affectée par les variations de $\delta$ et de $k_{\mathrm{V}}$. Des abaques ont été dressés par ces mêmes auteurs.

La détermination de la valeur du coefficient $k_{h}$ pour le dimensionnement d'un mur est, comme en stabilité dynamique des pentes, un problème non complètement résolu et pour lequel les choix sont en fait très arbitraires. Les valeurs de $k_{h}$ varient généralement de 0,1 à 0,3 . selon les pays, la zone sismique et les conditions de la fondation de l'ouvrage.

De très nombreux essais sur modèles réduits ont été effectués depuis 1929 et ont montré une assez bonne concordance entre la poussée maximale mesurée sur le parement et la poussée théorique calculée avec un coefficient sismique k égal à l'accélération maximale $\mathrm{a}_{\max }$ de la base. La liste complète des publications sur ces essais a été fournie par PRAKASH (1981). Cependant, dans une publication récente, AUBRY et CHOUVET (1985) ont montré par des calculs aux éléments finis reposant sur la loi sophistiquée de HUJEUX (1985), et utilisantl'accélérogramme mesuré à la base de leur modèle expérimental de SCHNEEBELI, que la théorie de MONONOBE$O K A B E$ pourrait très sensiblement sous-estimer l'effort dynamique maximal si la phase de plus forte sollicitation était précédée d'un certain nombre de phases de sollicitations moyennes ou faibles.

Il convient malgré tout d'être extrêmement prudent à l'égard des résultats quantitatifs fournis par les modèles réduits, qui ne respectent aucunement les lois de similitude du comportement dynamique des sols. Les effets de conditions aux limites, différentes de celles des ouvrages réels, s'ajoutent à ce non-respect des lois de similitude pour donner des coefficients d'amplification (rapport de l'accélération en tête à l'accélération de la base) supérieurs au cas des ouvrages réels comme le montre la note de M. BASTICK présentée à la suite de ce rapport général.

PRAKASH et SARAN (1966) ont proposé une extension de la méthode de MONONOBE-OKABE aux sols cohérents. Par analogie avec la poussée statique, ces 
auteurs expriment la poussée dynamique sous la forme suivante :

$$
\mathrm{P}_{\text {dyn }}=\gamma \mathrm{H}^{2} \cdot\left(\mathrm{N}_{\mathrm{a} \gamma}\right)_{\text {dyn }}+\mathrm{qH} \cdot\left(\mathrm{N}_{\mathrm{aq}}\right)_{\mathrm{dyn}}-\mathrm{cH} \cdot\left(\mathrm{N}_{\mathrm{ac}}\right)_{\text {dyn }}
$$

danslaquelle lescoefficients $\left(\mathrm{N}_{\mathrm{ay}}\right)_{\text {dyn }},\left(\mathrm{N}_{\mathrm{aq}}\right)_{\text {dyn }}$ et $\left(\mathrm{N}_{\mathrm{ac}}\right)_{\text {dyn }}$ dépendent de $\Phi, \gamma, \beta$ et $k$. Chacun de ces coefficients est exprimé par le rapport $\lambda_{i}$ de la valeur dynamique à la valeur statique. Le coefficient $\left(\mathrm{N}_{\mathrm{ac}}\right)_{\text {dyn }}$ a la même valeur que le coefficient statique correspondant qui, pour $\beta=0$, est égal à $2 \sqrt{\mathrm{Ka}}$. Les rapports $\lambda$, relatifs aux coefficients $\left(\mathrm{N}_{\mathrm{ar}}\right)_{\text {dyn }}$ et $\left(\mathrm{N}_{\mathrm{aq}}\right)_{\text {dyn }}$ sont très voisins de telle sorte qu'un seul rapport $\lambda$ est suffisant pour déterminer complètement la poussée dynamique maximale. Un abaque donnant les valeurs de $\lambda$ en fonction de l'angle de frottement interne $\Phi$ et du coefficient sismique $\mathrm{k}_{\mathrm{h}}$ est présenté à la figure 21 .

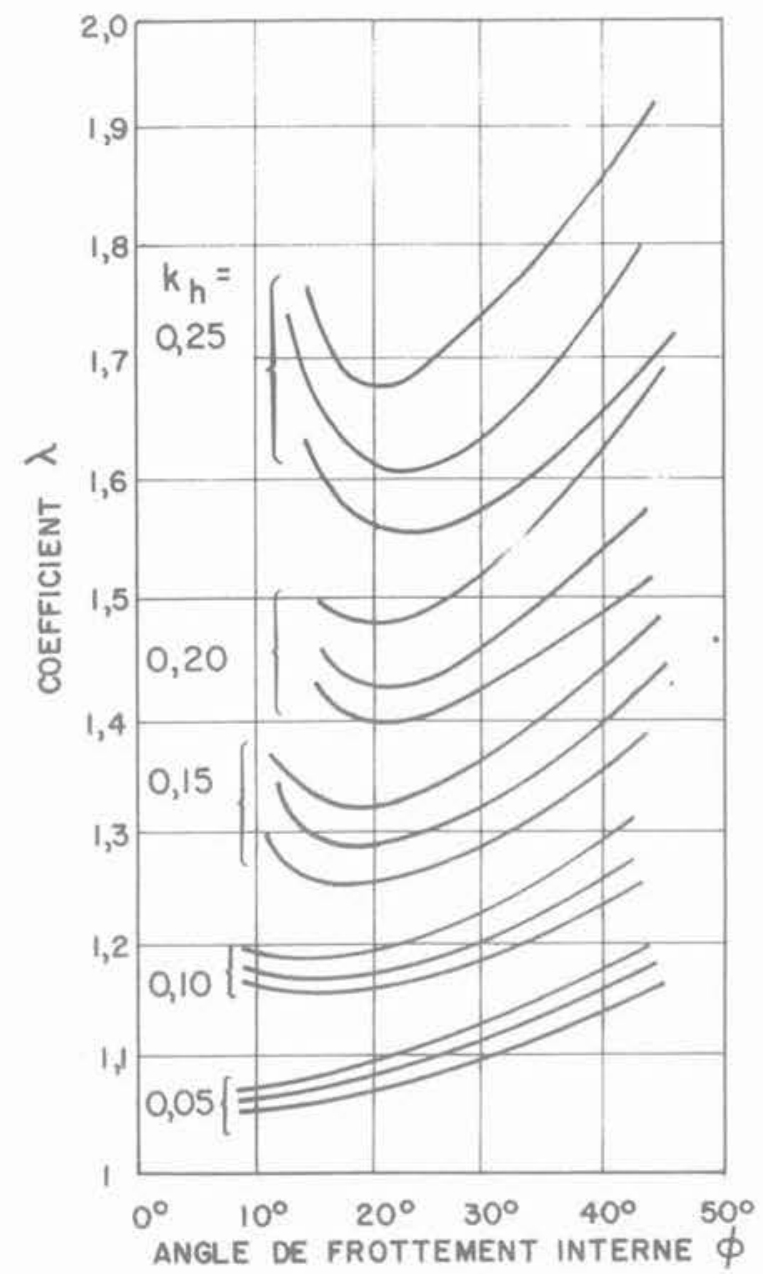

Fig. 21. - Abaque pour la prise en compte d'une cohésion du sol dans la méthode pseudostatique de Mononobe-Okabe (PRAKASH et SARAN, 1966).

Comme dans la théorie de Coulomb, la méthode de MONONOBE-OKABE calcule l'intensité de la poussée dynamique totale, mais ne donne aucune indication sur son point d'application. Cette question s'avère être très controversée dans la littérature. D'après une étude théorique de PRAKASH et BASAVANNA (1969), la hauteur du point d'application de la résultante au-dessus de la base passe de $0,4 \mathrm{H}$ à 0,5 Hlorsque le coefficient sismi-

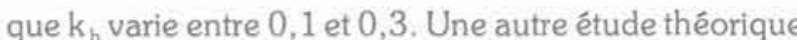
(WOODS, 1975), reposant sur l'hypothèse d'un sol élastique, indique un point d'application à mi-hauteur. L'étude par éléments finis de AUBRY et CHOUVET (1985) place ce point légèrement au-dessus de $\mathrm{H} / 3$.

Beaucoup d'auteurs et notamment la plupart des expérimentateurs séparent la poussée dynamique en une composante statique, correspondant à la poussée active classique, et un incrément dynamique. MATSUI (1941) et JACOBSEN (1951) indiquent, d'après les résultats de leurs essais, un point d'application de l'incrément dynamique à $2 \mathrm{H} / 3$ au-dessus de la base, valeur communément admise (SEED et WHITMAN, 1970). NANDKUMARAN (1973) indique que pour un mur flexible, le point d'application de cet incrément dynamique est plus haut que pour un mur rigide $(0,55 \mathrm{H}$ au lieu de $0,45 \mathrm{H})$

WHITMAN (1979) a montré que la rotation du mur avait une influence très grande sur la position du point d'application de la poussée dynamique. C'est ainsi que, durant les vibrations, ce point d'application varie généralement. Lorsque le mur ne s'incline pas et que le remblai se comporte plus ou moins élastiquement, la résultante passe dans la partie haute du mur. Mais dès que le mur commence à s'incliner vers l'extérieur, le point d'application de cette force descend dans le tiers inférieur. Lorsque la rotation se ralentit et s'arrête, la poussée dynamique repasse alors au-dessus du tiers inférieur et y reste jusqu'à la prochaine rotation vers l'extérieur.

Très peu de recherches et de publications concernent la butée dynamique. Physiquement, il est clair que la butée dynamique doit être inférieure à la butée statique. L'application de la méthode de MONONOBE-OKABE. avec un coefficient sismique vertical $k_{\text {v }}$ ascendant, donne une valeur de cette grandeur. Cependant, aucune expérimentation d'envergure n'a permis d'en vérifier la validité.

Toute la difficulté du dimensionnement d'un mur consiste à savoir quelle poussée prendre en compte dans le dimensionnement par rapport àla valeur maximale qui est développée au cours du séisme. La réponse est en grande partie fournie par les méthodes aux déplacements qui s'inspirent à nouveau de la méthode de NEWMARK.

\subsection{Méthode aux déplacements de RICHARDS et ELMS (1979)}

\subsubsection{Exposé de la méthode}

Considéré d'un point de vue strictement pseudostatique, le dimensionnement d'un mur de soutènement pourrait être réalisé de façon à ce que les forces motrices horizontales, tendant au déplacement du mur, soient inférieures ou égales aux forces résistantes, et qu'ainsi il n'y ait aucun déplacement du mur. RICHARDS et ELMS (1979) ont montré qu'un tel dimensionnement serait prohibitif, car la grande influence de l'inertie du mur conduit systématiquement à un déplacement, pour les ouvrages habituels soumis à un séisme. Il est ainsi nécessaire de développer un dimensionnement aux déplacements et, s'inspirant des travaux de NEWMARK, RICHARDS et ELMS ont pro- 
posé une méthode pour calculer un mur-poids aux séismes en fonction d'un déplacement de translation admissible. Ces auteurs se limitent à une cinématique de glissement sur la base. Ils étudient le mouvement du mur sous laction des forces dynamiques (poussée dynamique évaluée par la méthode de MONONOBE$O K A B E$, forces d'inertie), de son poids et du frottement sur le sol de fondation.

Comme dans la méthode de NEWMARK, le déplacement de translation du mur, occasionné par le séisme, est calculé par double intégration de l'accélérogramme de projet lorsque l'accélération de la base excède l'accélération limite k, g supportée sans glissement par le mur. Pour un mur usuel, la valeur de $k$, est de l'ordre de 0,1 .

A partir des travaux de FRANKLIN et CHANG (1977) qui ont exploité un grand nombre d'accélérogrammes réels, il est possible de calculer le déplacement du mur en fonction de $k_{\text {/, }}$, précédemment défini, de $a_{\max }$ et de $V$, respectivement accélération et vitesse maximales de la base au cours du séisme, par la formule empirique suivante, exprimée dans le Système International :

$$
\mathrm{d}=8,7 \cdot 10^{-4} \frac{\mathrm{V}^{2}}{\mathrm{a}_{\max }}\left[\frac{\mathrm{k}_{\ell} \cdot \mathrm{g}}{\mathrm{a}_{\max }}\right]^{-4}
$$

La procédure de dimensionnement proposée par RICHARDS et ELMS est alors la suivante :

$1^{\circ}$ On fixe la valeur $d_{\triangle}$ du déplacement admissible;

$2^{\circ}$ On déduit de la formule précédente la valeur $k_{l} . g$ de l'accélération que le mur doit supporter sans glissement ;

$3^{\circ}$ On calcule le poids du mur permettant de mobiliser un frottement sur la base suffisant pour équilibrer à la fois la poussée dynamique et la force d'inertie agissant sur le mur, calculées pour un coefficient sismique $\mathrm{k}_{f}$.

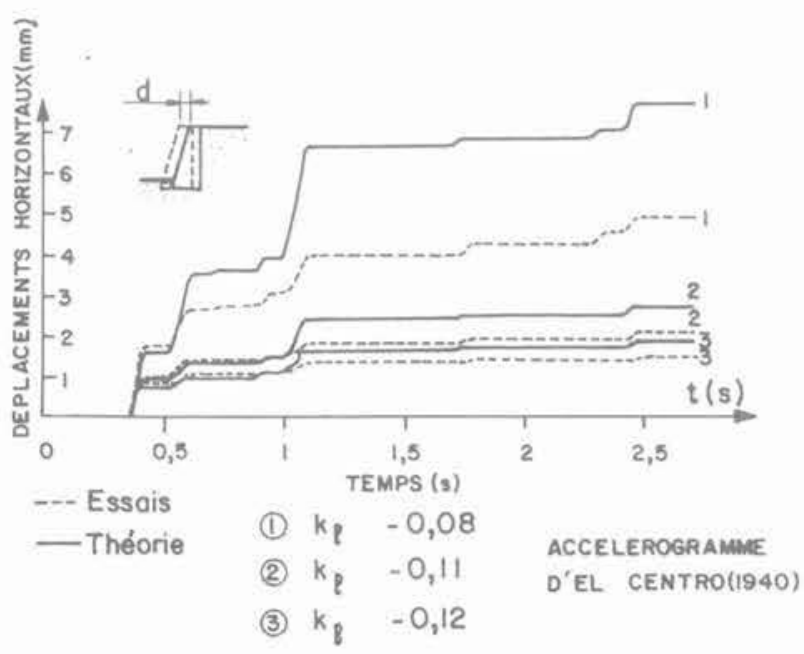

Fig. 22. - Comparaison entre les déplacements sismiques horizontaux de murs, calculés et mesurés en modèles réduits (LAl et BERRILL, 1979).
Autrement dit, cette méthode revient à dimensionner le mur pour qu'il y ait équilibre des forces dynamiques agissant sur lui au cours d'un séisme plus faible que le séisme de projet, séisme caractérisé par le coefficient sismique $k$, calculé en fonction du déplacement admissible $\mathrm{d}_{\mathrm{a}}$.

LAI et BERRILL (1979) ont réalisés des modèles réduits de mur qu'ils ont soumis, par l'intermédiaire d'une table vibrante, à des accélérogrammes réels. La figure 22 permet de comparer les déplacements mesurés à ceux prédits par la théorie de RICHARDS et ELMS pour trois murs-poids d'accélérations limites $k_{1} . \mathrm{g}$ différentes. Les déplacements calculés surestiment légèrement les déplacements mesurés, mais dans l'ensemble la concordance est satisfaisante, ce qui là encore doit être considéré avec prudence compte tenu des limitations déjà mentionnées des modèles réduits.

\subsubsection{Critiques et perfectionnements}

Cette méthode a le mérite de proposer une démarcho logique pour le dimensionnement des murs-poids sou $j$ séisme et a constitué, dans ce domaine, un progrès sensible. Cependant, elle reste schématique; aussi divers auteurs y ont-ils apporté des perfectionnements.

Critiquant l'hypothèse d'un comportement rigideplastique du sol, NADIM et WHITMAN (1983) ont montré que l'amplification du mouvement dans le remblai pouvait accentuer notablement l'amplitude des déplacements permanents du mur, si le rapport de la fréquence dominante de l'excitation de la base à la fréquence fondamentale du remblai excédait 0,3 . Ils ont proposé une amélioration simple de la méthode de RICHARDS et ELMS pour tenir compte de ce phénomène, consistant à majorer $\mathrm{V}$ et $\mathrm{a}_{\text {max }}$, vitesse et accélération maximales du séisme prises en compte dans le dimensionnement, en fonction de la valeur de ce rapport de fréquences.

ZARRABI (1979) a pris en considération l'accélération verticale du coin en glissement en arrière du mur, qui résulte de l'accélération horizontale de cette masse. Les modifications qu'il apporte à la méthode, conduisent à des déplacements légèrement plus faibles que ceux prévus par RICHARDS et ELMS.

Mais la principale limitation de la méthode de RICHARDS et ELMS résulte du caractère très restrictif de la cinématique retenue pour le mur (translation pure). En effet, à partir d'essais en modèle réduit de murs-poids, AUBRY et CHOUVET (1985) observent que la méthode de RICHARDS et ELMS ne donne des. résultats satisfaisants que pour les sollicitations modérées, tant que le mouvement de rotation reste faible. En revanche, pour des sollicitations plus fortes, la rotation ne peut plus être négligée et conduit à des déplacements de la tête du mur considérablement supérieurs à ceux prévus par le calcul de RICHARDS et ELMS. Une meilleure prise en compte de la complexité de la cinématique semble donc nécessaire.

En fait, la prédiction de la rotation du mur lors du séisme constitue une tâche difficile que peu d'auteurs ont abordée jusqu'à ce jour. PRAKÁSH et al. (1981) découplent le mouvement de rotation de celui de 
translation et écrivent l'équation des moments autour de l'arête interne de la base du mur, équation qu'ils résolvent numériquement. Les difficultés majeures de ce type d'approche consistent à évaluer correctement la contribution des forces dynamiques de poussée et de butée, conditionnée en particulier par la position des points d'application de ces forces, et celle de la réaction du mur sur le sol de fondation.

\subsection{Cas particuliers des murs en terre armée}

\subsubsection{Phénomènes dynamiques dans les murs en terre armée}

On rencontre de nombreux ouvrages en terre armée dans les zones de forte séismicité, d'une part en raison de l'expansion considérable qu'a prise cette technique, et d'autre part en raison des performances très satisfaisantes de ce type de structures vis-à-vis de la sollicitation sismique. Aussi des recherches importantes ontelles été entreprises pour affiner la compréhension du comportement dynamique des murs en terre armée et déboucher sur des méthodes de dimensionnement tenant compte de leurs spécificités.

Comme pour les autres types d'ouvrages de soutènement soumis à des séismes, le dimensionnement doit prendre en compte le risque de glissement sur la base sous l'action de la poussée dynamique du remblai. Mais dans les murs en terre armée, le séisme peut également mettre en cause la stabilité interne de l'ouvrage en affectant directement la structure même du mur. La compréhension de ce phénomène est donc de toute première importance.

Les premiers pas dans cette direction ont été faits par RICHARDSON et LEE (1974), qui ont étudié qualitativement le comportement de modèles réduits de murs en terre armée à la table vibrante. Ils ont mis en évidence deux effets majeurs du séisme sur ces ouvrages.
La sollicitation sismique augmente les tractions maximales dans les armatures qui peuvent casser au cours du chargement. On observe alors une rupture brutale de l'ouvrage. On constate également une diminution de l'adhérence terre-armature pouvant entraîner des déplacements importants du parement quil s'arrêtent à la fin de la sollicitation.

Ces deux effets défavorables de la sollicitation sismique se superposent pour diminuer la stabilité interne du mur. Comme on l'a vu, en raison du non-respect des lois de similitude, les essais à la table vibrante en modèles réduits ne peuvent malheureusement pas fournir d'indications quantitatives sur ces phénomènes. La connaissance provient donc d'essais en vraie grandeur, de modèles faiblement réduits et de calculs numériques en éléments finis. On présente dans la suite les principaux acquis des recherches dans ce domaine et leur application aux méthodes de dimensionnement, dont on trouvera une étude plus détaillée dans BASTICK et SCHLOSSER (1985).

\subsubsection{Stabilité interne d'un mur en terre armée sous séisme}

\section{EFFET D'UN SÉISME SUR LES TRACTIONS DANS LES ARMATURES}

La connaissance des efforts dynamiques s'exerçant sur les armatures au cours du séisme est essentielle pour l'évaluation des risques de rupture par cassure ou défaut d'adhérence.

CHIDA (1980) a réalisé de remarquables essais à la table vibrante sur des modèles de murs en terre armée à l'échelle $1 / 2$ dans une gamme de fréquences de 2 à $7 \mathrm{~Hz}$ qui ont permis de juger des modifications quantitatives apportées par un séisme à la distribution statique des tractions en fonction de la distance au parement. Les résultats ainsi qu'un schéma du modèle utilisé sont présentés à la figure 23 .
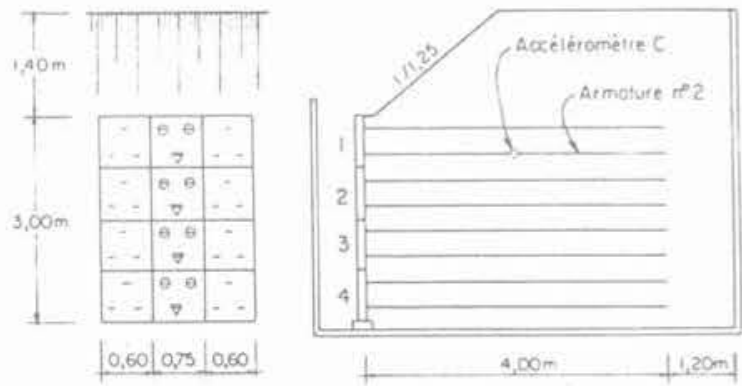

Fig. 23a. - Modèle à l'échelle 1/2 sur table vibrante.

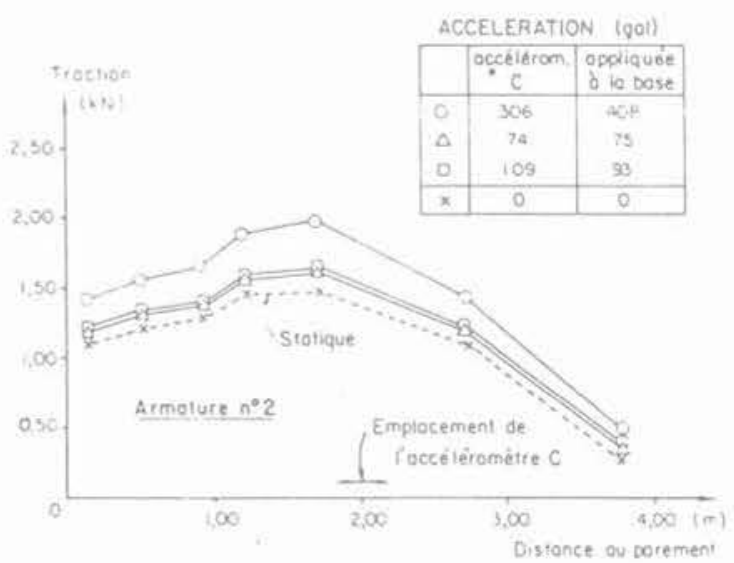

Fig. 23b. - Traction mesurée dans l'armature $n^{\circ} 2$ pour divers cas de charges dynamiques. 
Au cours de la sollicitation cyclique, on constate que les tractions de plus forte intensité sont obtenues en superposant aux valeurs statiques un incrément dynamique variant peu avec la distance au parement et dont le maximum se situe au voisinage de $0,5 \mathrm{H}$. Le lieu des tractions maximales qui délimite la zone active à prendre en compte en dynamique se trouve ainsi légèrement repoussé en arrière. On considère généralement une variation affine de la largeur D de cette zone en fonction de l'accélération horizontale maximale $\mathrm{a}_{\max }$ selon la formule :

$$
D=\left(0,3+a_{\max } / 2 g\right) \cdot H
$$

qui redonne en particulier la valeur classique $\mathrm{D}=0,3 \mathrm{H}$ dans le cas statique.

Des calculs numériques en éléments finis à l'aide du programme SUPERFLUSH ont permis de retrouver les résultats expérimentaux de CHIDA. Une description détaillée de SUPERFLUSH est présentée dans la note de $\mathrm{M}$. BASTICK jointe à ce rapport général.

Pour le calcul des tractions dynamiques maximales, on prend en compte une force pseudostatique égale à la valeur maximale de la force d'inertie agissant sur la nouvelle zone active, ce qui conduit à la formule :

$$
E_{1}^{\prime}=0,225 \gamma H^{2} a_{\max } / g\left(1+5 / 3 \cdot a_{\max } / g\right)
$$

\section{FROTTEMENT DYNAMIQUE TERRE-ARMATURE}

En 1979, MURRAY et al. ont expliqué la réduction de ce coefficient dans le cas d'armatures lisses par la diminution de la contrainte verticale instantanée s'exerçant sur ces armatures provoquée par les ondes d'accélération verticale. L'exploitation des résultats du mur expérimental de Millville en Virginie (1983) a permis de quantifier cette perte d'adhérence dans le cas d'armatures nervurées. Le rapport $\mu_{d}^{*} / \mu_{s}^{*}$ du coefficient de frottement apparent dynamique au coefficient de frottement apparent statique variait très peu autour de 0,8

\subsubsection{Dimensionnement externe d'un mur en terre armée sous séisme}

L'analyse de la stabilité externe sous séisme des murs en terre armée qui implique la prise en compte dans l'étude de l'équilibre du mur de l'interaction dynamique entre le mur, sa base et le remblai est restée très classique et simplifiée. Elle se limite à un calcul pseudostatique.

Comme dans le cas d'un mur classique, il est nécessaire de faire intervenir une poussée dynamique sur le mur supérieure à la poussée statique initiale. Elle est calculée par la formule de MONONOBE-OKABE, simplifiée par SEED et WHITMAN (1970) sous la forme :

$$
\left(P_{a}\right)_{d y n}=0,375 \gamma \mathrm{H}^{2} \cdot \frac{a_{\max }}{\mathrm{g}} \text {. }
$$

Les murs en terre armée mettant en jeu une masse considérable, la force d'inertie agissant sur l'ouvrage de soutènement joue un rôle essentiel et même prépondérant. Cependant, elle est difficile à évaluer, car son intensité dépend dans une large mesure de la flexibilité du massif armé.

En raison de cette flexibilité, la force d'inertie et la poussée dynamique à l'arrière du mur n'atteignent pas simultanément leurs maxima au cours de la sollicitation sismique, ce qui complique sensiblement le calcul pseudostatique. Dans un document interne de la société Terre Armée, SEED a proposé de retenir la valeur suivante pour la somme $S_{\text {dyn }}$ de ces actions :

$$
\mathrm{S}_{\text {dyn }}=0,7 \gamma \mathrm{H}^{2} \mathrm{a}_{\max } / \mathrm{g} \text {. }
$$

\subsubsection{Comportement des ouvrages réels}

Il n'existe pas d'exemple d'ouvrage en terre armée qui, soumis à un tremblement de terre, ait subi des désordres notables. Le cas du séisme de Liège (Belgique) en 1983, caractérisé par des accélérations maximales $\mathrm{a}_{\max }$ de $0,15 \mathrm{~g}$ à $0,2 \mathrm{~g}$ est particulièrement frappant. En comparant des mesures réalisées peu de temps avant le séisme et après celui-ci, on a pu vérifier que les déplacements subis par le mur de Jemeppe $(\mathrm{H} \simeq 6 \mathrm{~m})$, placé à quelques kilomètres de l'épicentre, restaient inférieurs à $5 \mathrm{~mm}$. A titre de comparaison, la dalle supérieure d'un garage situé à proximité s'était déplacée de plusieurs centimètres.

Par ailleurs, il est remarquable que ce mur n'avait pas fait l'objet d'un calcul au séisme. Les coefficients de sécurité utilisés pour un dimensionnement statique paraissent donc suffisants pour se prémunir contre les effets d'un séisme dont l'accélération maximale n'excède pas $0,2 \mathrm{~g}$.

\section{CONCLUSION}

Dans la réponse des pentes et des ouvrages de soutènement aux séismes, la nature dynamique de la sollicitation joue un rôle essentiel. Elle met en jeu des phénomènes spécifiques, dont l'étude en laboratoire et la prise en compte dans le dimensionnement requièrent des traitements souvent plus complexes que dans le cas statique. L'une et l'autre ont fait des progrès considérables durant les vingt dernières années. L'état des connaissances qui vient d'être dressé permet de dégager les conclusions suivantes :

\subsection{Comportement dynamique des sols}

5.1.1. La plupart des aspects majeurs du comportement dynamique des sols semblent avoir été mis en évidence. Cependant, certains points importants restent à approfondir.

5.1.2. Dans le domaine des sols grenus, le cas particulier des sables effondrables met en jeu le phénomène de déformation d'écoulement dont les effets peuvent être aussi redoutables que ceux de la liquéfaction cyclique. Une méthode de caractérisation in situ de ce type de matériau fait actuellement défaut.

5.1.3. Toujours pour les sables, les travaux récents de VAID, CHERN et FINN ont permis de clarifier la question controversée de l'influence d'un cisaillement initial 
sur la résistance au cisaillement cyclique non drainée. Comme le montrent ces auteurs, les résultats des recherches effectuées dans ce domaine concordent, à condition de comparer la réponse de matériaux de même densité.

5.1.4. Dans le domaine des sols fins, le comportement des argiles normalement consolidées sous chargement cyclique présente des analogies marquées avec celui des sables. En particulier, on retrouve la validité de la courbe intrinsèque déterminée en statique et le rôle prépondérant dés surpressions interstitielles. En revanche, les argiles fortement surconsolidées semblent présenter un comportement à la rupture du type fatigue, encore incomplètement clarifié.

5.1.5. Pour les argiles, comme pour les sables, la prévision des surpressions interstitielles reste délicate car les paramètres qui influent sur la génération et la dissipation de ces dernières sont nombreux. De plus, une solution rigoureuse de ce problème doit prendre en compte le couplage volumique qui existe entre le squelette solide et le fluide interstitiel. Aussi la plupart des critères de résistance utilisés reposent-ils sur une analyse de la réponse dynamique en contraintes totales.

\subsection{Comportement des ouvrages (modèles réduits)}

Plus encore qu'en statique, le non-respect des lois de similitudes réduit considérablement le volume d'informations qu'il est possible de tirer des essais en modèles réduits. Il faut être prudent et se contenter d'indications qualitatives, ce qui fait que la validité d'une méthode de dimensionnement donnée ne peut en général être testée que par des essais sur modèles faiblement réduits ou par des observations sur des ouvrages réels.

\subsection{Dimensionnement des ouvrages}

5.3.1. Les études phénoménologiques réalisées sur échantillons homogènes de sols ou sur modèles réduits d'ouvrages ont permis peu à peu l'élaboration de méthodes de dimensionnement prenant en compte le caractère dynamique de la sollicitation et devant, à terme, remplacer les méthodes pseudostatiques.

5.3.2. Dans le domaine des pentes, les approches inspirées de la méthode du chemin de contraintes ainsi que les méthodes de seuil se sont avérées très fructueuses, à la fois pour la prévision des déplacements provoqués dans le massif par le séisme et pour la prise en compte du risque de liquéfaction. Ces méthodes ont permis d'interpréter qualitativement et quantitativement le comportement d'ouvrages réels ayant été soumis à des séismes dans des cas où la méthode pseudostatique s'était avérée inadaptée.

Ces analyses restent néanmoins simplifiées. Le calcul des contraintes est le plus souvent effectué au moyen de lois de comportement (par exemple viscoélasticité linéaire équivalente) ne prenant en compte que certains aspects du comportement réel. En particulier, la prévision des déplacements et l'évaluation du risque de liquéfaction sont découplées du calcul des contraintes, ce qui demeure physiquement peu satisfaisant.

5.3.3. Dans le domaine des ouurages de soutènement, peu de recherches ont été effectuées en dehors des murs-poids et des murs en terre armée.

De façon générale, analyses théoriques et essais en modèles réduits ont mis en évidence le rôle essentiel de l'inertie de l'ouvrage sur l'amplitude des déplacements provoqués par le séisme. Des méthodes de calcul prenant en compte la cinématique de translation par à-coups d'un mur-poids sous séisme ont été développées pour dimensionner ce type d'ouvrage à partir de la donnée arbitraire d'une translation admissible. Il faut déplorer que peu d'auteurs aient tenté de prendre en compte une cinématique plus complète incluant la possibilité de rotation du mur.

Les recherches sur les murs en terre armée ont permis d'étudier, et de prendre en compte dans le dimensionnement, la stabilité interne de la structure, ce qui s'avère un élément essentiel pour tous les types d'ouvrages de soutènement complexes.

\section{BIBLIOGRAPHIE}

ANDERSEN K.H. (1976), Behaviour of Clay Subjected to Undrained Cyclic Loading, First International Conference on Behaviour of Offshore Structures, vol. I, pp. 392-403.

AUBRY D., CHOUVET D. (1985), Calcul Sismique des Murs de Soutènement, Génie Parasismique, chap. VIII-3, Presses de l'E.N.P.C., Paris.

BASTICK M., SCHLOSSER F. (1986), Comportement et Dimensionnement Dynamiques des Ouvrages en Terre Armée, Ier Colloque National de Génie Parasismique, Association Française du Génie Parasismique.

BHATIA S. (1980), The Verification of Relationships for Effective Stress Method to Evaluate Liquefaction Potential of Saturated Sands, Ph. D. Thesis, Department of Civil Engineering, University of British Columbia, Vancouver.

CASTRO G. (1975), Liquefaction and Cyclic Mobility of Saturated Sands, Journal of the Geotechnical Engineering Division, ASCE, vol. Cl, ${ }^{\circ}$ GT 6 .

CHIDA S., MINAMI K., ADACHI K. (1982), Test de stabilité de remblais en Terre Armée, Publication interne du ministère des Travaux Publics Japonais. FINN W.D.L. (1981), Liquefaction Potential : Developments since 1976, International Conference on Recent Advances in Geotechnical Earthquake Engineering and Soil Dynamics, Rolla, Missouri, pp. 655-679.

FRANKLIN A.G., CHANG F.K. (1977), Earthquake Resistance of Earth and Rock-Fill Dams, Report 5 : Permanent Displacements of Earth Embankments by Newmark Sliding Block Analysis, Misc. Paper S-71.17, Soils and Pavements Laboratory, U.S. Army Engineer Waterways Experiment Station, Vicksburg, Missouri. 
GHABOUSSI J., DIKMAN S.U. (1978), Liquefaction Analysis of Horizontally Layered Sands, Journal of the Geotechnical Engineering Division, ASCE, vol. CIV, $n^{\circ}$ GT 3 .

GOODMAN R.E., SEED H.B. (1966), Earthquakeinduced Displacements in Sand Embankments, Journal of the Soil Mechanics and Foundations Division, vol. XCII, n SM2.

HICHER P.Y. ELHOSRI M.S. (1981), Cyclic Loading on Clays, International Conference on Recent Advances in Geotechnical Earthquake Engineering and Soil Dynamics, Rolla, Missouri, pp. 645-648.

HUJEUX J.C. (1985), Un Modèle Élastoplastique pour les Sols, Génie parasismique, chap. IV.2, Presses de l'E.N.P.C., Paris.

JACOBSEN L.S. (1951), Kentucky Project Report $n^{\circ} 13$, T.V.A. Series 1951, Appendix D.

KONING H.L. (1963), Some Observations on the Modulus of Compressibility of Water, VIIle Conférence Européenne de Mécanique des Sols et des Travaux de Fondation, Helsinki, vol. I, pp. 33-36.

KROEZEN M., VELLINGA P., LINDENBERG J., BURGEN A.M. (1982), Geotechnical and Hydraulic Aspects with Regard to Seabed and Slope Stability, 2nd Canadian Conference on Marine Geotechnical Engineering, Halifax, Canada.

LAI CHO SIM, BERRILL J.B. (1979), Shaking Table Tests on a Model Retaining Wall, South Pacific Regional Conference on Earthquake Engineering, Wellington, New Zeland.

LEE K.L., SEED H.B. (1967), Dynamic Strength of Anisotropically Consolidated Sand, Journal of Soil Mechanics and Foundations Division ASCE, vol. XCIII, $n^{\circ}$ SM5.

LEE K.L. (1974), Seismic Permanent Deformations in Earth Dams, Report n ${ }^{\circ}$ UCLA-ENG-7497, School of Engineering and Applied Science, University of California, Los Angeles.

LEE K.L., Roth W. (1977), Seismic Stability Analysis of Hawkins Hydraulic Fill Dam, Journal of the Geotechnical Engineering Division, ASCE, vol. CIII, $n^{\circ}$ GT 6.

LUONG M.P. (1980), Phénomènes Cucliques dans les Sols Pulvérulents, Revue Française de Géotechnique, $n^{\circ} 10$.

MAKDISI F.I., SEED H.B. (1978), Simplified Proce. dure for Estimating Dam and Embankment Earthquake-Induced Deformations, Journal of the Geotechnical Engineering Division, ASCE, vol. CIV, $n^{\circ}$ GT 7 .

MARTIN G.R., FINN W.D.L., SEED H.B. (1975), Fundamentals of Liquefaction Under Cyclic Loading, Journal of the Geotechnical Engineering Division, ASCE, vol. CI, n GT 5 .

MARTIN G.R., FINN W.D.L., SEED H.B. (1978), Effects of System Compliance on Liquefaction Tests, Journal of the Geotechnical Engineering Division, ASCE, vol. CIV, n GT 4.

MATSUI H. (1941), Experimental Study on the Distribution of Earth Pressure Acting on a Vertical Wall During Earthquakes, Journal of the Japanese Society of Civil Engineers, vol. XXVII, $n^{\circ} 2$.

MATSUI T., OHARA H., ITO T. (1980), Cyclic Stress-Strain History and Shear Characteristics of Clay, Journal of the Geotechnical Engineering Division, ASCE, vol. CVI, n GT 10 .

MATSUI T., ABE N. (1981), Behaviour of Clay on
Cyclic Stress-Strain History, $\mathrm{X}^{\mathrm{e}}$ Conférence Internationale de Mécanique des Sols et de Travaux de Fondations, Stockholm, pp. 261-264.

MOKHAM M. (1983), Contribution à l'Étude Expérimentale et Théorique du Comportement des Sables sous Chargements Cycliques, thèse de Docteur Ingénieur de l'Université de Grenoble.

MONONOBE N., MATSUO H. (1929), On the Determination of Earth Pressures During Earthquakes, World Engineering Conference, vol. IX, p. 176.

MURPHY V.A. (1960), The Effect of Ground Characteristics on the Aseismic Design of Structures. 2nd World Conference on Earthquake Engineering, Tokyo, Japan.

MURRAY R.T., CARTER D.R., KRAWCZYK J.V. (1979), Pull-out Tests on Reinforcement Embedded in Uniformly Graded Sand Subjected to Vibration, VIIe Conférence Européenne de Macanique des Sols, Brighton, vol. III, pp. 115-120.

NADIM F., WHITMAN R.V. (1983), Seismically Induced Movement of Retaining Walls, Journal of the Geotechnical Engineering Division, ASCE, vol. CIX, n GT 7

NANDKUMARAN P. (1973), Behaviour of Retaining Walls Under Dynamic Loads, Ph. D. Thesis, Roorkee University, Roorkee, India.

NAZARIAN H.N., HADJIAN A.H. (1979), Earthquake Induced Lateral Soil Pressures on Structures, Journal of the Geotechnical Engineering Division, ASCE, vol. CV, n GT9.

NEWMARK N.M. (1965), Effects of Earthquakes on Dams and Embankments, Ve Conférence Rankine, Géotechnique, vol. XV, n²

POST G., FLORENTIN P. (1981), Conception Parasismique des Barrages en Remblais, Revue Travaux, $\mathrm{n}^{\circ} 553$.

PRAKASH S., SARAM S. (1966), Static and Dynamic Earth Pressures Behind Retaining Walls, 3rd Symposium on Earthquake Engineering, University of Roorkee, Roorkee, India, vol. I, pp. 277-288.

PRAKASH S., BASAVANNA B.M. (1969), Earth Pressure Distribution Behind Retaining Walls During an Earthquake, 4th World Conference on Earthquake Engineering, Santiago, Chili.

PRAKASH S. (1981), Analysis of Rigid Retaining Walls During Earthquakes, International Conference on Recent Advances in Geotechnical Earthquake Engineering and Soil Dynamics, Rolla, Missouri, pp. 9931020.

PRAKASH S., PURI V.K., KHANDOKER J.U. (1981), Displacement Analysis of Rigid Retaining Walls in Rocking, International Conference on Recent Advances in Geotechnical Earthquake Engineering and Soil Dynamics, Rolla, Missouri, pp. 1021-1025. RICHARDS R., ELMS D.G. (1979), Seismic Behaviour of Gravity Retaining Walls, Journal of the Geotechnical Engineering Division, ASCE, vol. CV, $n^{\circ}$ GT 4.

RICHARDSON G.N., LEE K.L. (1974), Seismic Design of Reinforced Earth Walls, ASCE, Nat. Meeting on Wat. Resources Eng., L.A. California.

SANGREY D.A., HENKEL D.J., ESRIG M.I. (1969), The Effective Stress Response of a Saturated Clay Soil to Repeated Loading, Canadian Geotechnical Journal, vol. VI, $\mathrm{n}^{\circ} 3$.

SEED H.B. (1966), A Method for Earthquake Resistant Design of Earth Dams, Journal of the Soil Mecha- 
nics and Foundations Division, ASCE, vol. XCII, $n^{\circ}$ SM 1.

SEED H.B., MARTIN G.R. (1966), The Seismic Coefficient in Earth Dam Design, Journal of the Soil Mechanics and Foundations Division, ASCE, vol. XCII, $n^{\circ} \mathrm{SM} 3$.

SEED H.B., CHAN C.K. (1966), Clay Strength Under Earthquake Loading Conditions, Journal of the Soil Mechanics and Foundations Division, ASCE, vol. XCII, $n^{\circ}$ SM2.

SEED H.B., LEE K.L., IDRISS I.M. (1969), Analysis of the Sheffield Dam Failure. Journal of the Soil Mechanics and Foundations Division, ASCE, vol. XCV, $n^{\circ} \mathrm{SM} 6$.

SEED H.B. WHITMAN R.V. (1970), Design of Earth Retaining Structures for Dynamics Loads, Lateral Stresses in the Ground and Earth Retaining Structures, ASCE.

SEED H.B., IDRISS I.M., LEE K.L. (1975), Dynamic Analysis of the Slide in the Lower San Fernando Dam During the Earthquake of February 9, 1971, Journal of the Geotechnical Engineering Division, ASCE, vol, CI, $n^{\circ}$ GT 9

SEED H.B. (1979a), Considerations in the Earthquake-Resistant Design of Earth and Rockfill Dams, XIXe Conférence Rankine, Géotechnique, vol. XXIX, n 3 .

SEED H.B. (1979b), Soil Liquefaction and Cyclic Mobility Evaluation for Level Ground During Earthquakes, Journal of the Geotechnical Engineering Division, ASCE, vol. CV, n GT 2.

SEED H.B. (1981), Earthquake-Resistant Design of Earth Dams, International Conference on Recent Advances in Soil Dynamics and Earthquake Engineering, Rolla, Missouri.

SERFF N., SEED H.B., MAKDISI F.I., CHANG C.K. (1976), Earthquake Induced Deformations of Earth Dams, Report $n^{\circ}$ EERC 76-4, Earthquake Engineering Research Center, University of California, Berkeley. SLADEN J.A., D'HOLLANDER R.D., KRAHN J. (1985), The Liquefaction of Sands, a Collapse Surface Approach, Canadian Geotechnical Journal, vol. XXII, $\mathrm{n}^{\circ} 4$.
TAKAHASHI M., HIGHT D.W., VAUGHAN P.R. (1980), Effective Stress Changes observed During Undrained Cyclic Triaxial Tests on Clay, International Symposium on Soils Under Cyclic and Transient Loading, Swansea.

TANIGUCHI E., SASAKI Y. (1985), Back Analysis of a Landslide due to the Naganoken Seibu Earthquake of September 14, 1984, Specialty Session on Seismic Stability of Slopes, 11th ICSMFE, San Francisco.

TERZAGHI K. (1950), Mechanisms of Landslides, The Geological Survey of America, Engineering Geology (Berkley).

VAID P.Y., FINN W.D.L. (1979), Static Shear and Liquefaction Potential, Journal of the Geotechnical Engineering Division, ASCE, vol. CV, nº GT 10.

VAID P.Y., CHERN J.C. (1983), Effect of Static Shear on Resistance to Liquefaction, Soils and Foundations, vol. XXIII, $n^{\circ} 1$.

WHITMAN R.V. (1979), Dynamic Behaviour of Soils and its Application to Civil Engineering Projects, VIe Conférence Panaméricaine de Mécanique des Sols et de Travaux de Fondations, vol. 1, pp. 59-105.

WILSON N.E., GREENWOOD J.R. (1974), Pore Pressures and Strains after Repeated Loading of Saturated Clay, Canadian Geotechnical Journal, vol. XI, $n^{\circ} 2$.

WOODS J.H. (1975), Earthquake-Induced Soil Pressures on Structures, Report $n^{\circ}$ EERL-73-05, Earthquake Engineering Research Laboratory, California Institute of Technology, Pasadena.

WOOD D.M. (1976), Comments on Cyclic Loading on Clay, First International Conference on Behaviour of Offshore Structures, vol. I, pp. 418-424.

YZIQUEL A., LINO M., POST G., TARDIEU B. (1981), Seismic Analysis as a Tool in the Design of Two Earth Dams, Symposium on Advances in Geotechnical Earthquake Engineering, Saint-Louis, Missouri.

ZARRABI K. (1979), Sliding of Gravity Retaining Wall During Earthquake Considering Vertical Acceleration and Changing Inclination of Failure Surface, S.M. Thesis, Department of Civil Engineering, M.I.T., Cambridge, Massachussets. 